\title{
A TRPC5-regulated calcium signaling pathway controls dendrite patterning in the mammalian brain
}

\author{
Sidharth V. Puram, ${ }^{1,2}$ Antonio Riccio, ${ }^{1,4}$ Samir Koirala, ${ }^{5}$ Yoshiho Ikeuchi, ${ }^{1}$ Albert H. Kim,,${ }^{1,6}$ \\ Gabriel Corfas, ${ }^{3,5}$ and Azad Bonni ${ }^{1,2,7}$ \\ ${ }^{1}$ Department of Neurobiology, ${ }^{2}$ Program in Biological and Biomedical Sciences, ${ }^{3}$ Department of Otolaryngology, Harvard Medical \\ School, Boston, Massachusetts 02115, USA; ${ }^{4}$ Department of Cardiology, Manton Center for Orphan Disease, ${ }^{5}$ F.M. Kirby \\ Neurobiology Center, 'Department of Neurosurgery, Brigham and Women's Hospital, Children's Hospital, Boston, \\ Massachusetts 02115, USA
}

\begin{abstract}
Transient receptor potential (TRP) channels have been implicated as sensors of diverse stimuli in mature neurons. However, developmental roles for TRP channels in the establishment of neuronal connectivity remain largely unexplored. Here, we identify an essential function for TRPC5, a member of the canonical TRP subfamily, in the regulation of dendrite patterning in the mammalian brain. Strikingly, TRPC5 knockout mice harbor long, highly branched granule neuron dendrites with impaired dendritic claw differentiation in the cerebellar cortex. In vivo RNAi analyses suggest that TRPC5 regulates dendrite morphogenesis in the cerebellar cortex in a cell-

autonomous manner. Correlating with impaired dendrite patterning in the cerebellar cortex, behavioral analyses reveal that TRPC5 knockout mice have deficits in gait and motor coordination. Finally, we uncover the molecular basis of TRPC5's function in dendrite patterning. We identify the major protein kinase calcium/calmodulindependent kinase II $\beta$ (CaMKII $\beta$ ) as a critical effector of TRPC5 function in neurons. Remarkably, TRPC5 forms a complex specifically with CaMKII $\beta$, but not the closely related kinase CaMKII $\alpha$, and thereby induces the CaMKII $\beta$-dependent phosphorylation of the ubiquitin ligase Cdc20-APC at the centrosome. Accordingly, centrosomal CaMKII $\beta$ signaling mediates the ability of TRPC5 to regulate dendrite morphogenesis in neurons. Our findings define a novel function for TRPC 5 that couples calcium signaling to a ubiquitin ligase pathway at the centrosome and thereby orchestrates dendrite patterning and connectivity in the brain.
\end{abstract}

[Keywords: cerebellar cortex; dendrites; protein kinase signaling]

Supplemental material is available for this article.

Received July 8, 2011; revised version accepted November 3, 2011.

The regulation of dendrite development is essential for the establishment of neuronal circuits in the brain (Hausser et al. 2000; Jan and Jan 2003; Grueber and Jan 2004; de la Torre-Ubieta and Bonni 2011). Beyond providing a better understanding of brain development, studies of dendrite morphogenesis have garnered increasing interest because disturbances in dendrite structure are featured in diverse neurological diseases, including mental retardation and autism spectrum disorders (Kaufmann and Moser 2000; Dierssen and Ramakers 2006; Pardo and Eberhart 2007).

Calcium signaling plays a central role in the regulation of dendrite morphogenesis and connectivity in the developing brain (Wong and Ghosh 2002). Voltage-sensitive

${ }^{7}$ Corresponding author.

E-mail azad_bonni@hms.harvard.edu.

Article published online ahead of print. Article and publication date are online at http://www.genesdev.org/cgi/doi/10.1101/gad.174060.111. calcium channels (VSCCs) and NMDA receptors provide a major mode of calcium entry in neurons and trigger downstream signaling cascades that control dendrite development (Konur and Ghosh 2005; Cline and Haas 2008). However, the role of other calcium channels in the regulation of dendrite development remains largely to be elucidated. In particular, the potential role of the large family of transient receptor potential (TRP) channels in dendrite morphogenesis and connectivity remains unexplored.

TRP channels have been characterized as cellular sensors of diverse stimuli, including temperature, nociception, taste, and mechanical forces (McKemy et al. 2002; Montell et al. 2002; Clapham 2003; Ramsey et al. 2006; Talavera et al. 2008). Recent data suggest that TRP channels might also harbor developmental functions in neurons. Members of the canonical TRP (TRPC) channel subfamily, which are highly expressed in the developing mammalian brain, have been implicated in the control of growth cone morphology and responses in vitro /Greka 
et al. 2003; Davare et al. 2009), raising the intriguing possibility that TRPC channels might contribute to the regulation of neuronal morphogenesis and connectivity.

Calcium entry through VSCCs is thought to act via transcriptional mechanisms to promote dendrite growth and branching (Redmond et al. 2002; Gaudilliere et al. 2004), while calcium entry through NMDA receptors may act locally to control dendrite branching and stabilization (Rajan et al. 1999; Sin et al. 2002). Recent studies have identified the centrosome as a critical signaling hub that regulates dendrite morphogenesis (Kim et al. 2009). The major mitotic E3 ubiquitin ligase Cdc20-APC operates at the centrosome to promote dendrite growth and arborization. These studies have raised the question of whether calcium might regulate ubiquitin signaling at the centrosome.

The protein kinase calcium/calmodulin-dependent protein kinase II (CaMKII) represents a major target of calcium signaling downstream from VSCCs and NMDA receptors in neurons (Wu and Cline 1998; Rajan et al. 1999; Vaillant et al. 2002; Gaudilliere et al. 2004). Intracellular calcium binds to calmodulin (CaM), which activates CaMKs, including CaMKII. In the mammalian brain, CaMKII predominantly consists of the $\alpha$ and $\beta$ isoforms (Miller and Kennedy 1985; Vallano 1989; Kanaseki et al. 1991), which have divergent functions in dendrite patterning. CaMKII $\alpha$ is activated in response to calcium entry via VSCCs and subsequently phosphorylates the transcription factor NeuroD at Ser336 and thus stimulates dendrite growth and elaboration (Gaudilliere et al. 2004). In contrast, CaMKII $\beta$ phosphorylates the APC ubiquitin ligase coactivator Cdc20 at Ser51 and thereby inhibits centrosomal Cdc20-APC activity and consequently triggers dendrite retraction and pruning (Puram et al. 2011). However, the fundamental question of the identity of calcium channels operating upstream of CaMKII $\beta$ in the control of dendrite patterning remained to be addressed.

In this study, we discovered an essential function for the channel TRPC5 as a critical regulator of dendrite patterning in the mammalian brain. TRPC5 knockout mice harbor exuberant granule neuron dendrite arbors with deficient post-synaptic dendritic claw differentiation in the cerebellar cortex. In vivo RNAi electroporation analyses show that TRPC5 controls dendrite patterning in the cerebellar cortex in a cell-autonomous manner. Behavioral analyses reveal that TRPC5 knockout mice have deficits in gait and motor coordination. Finally, we identified a mechanism by which TRPC5 regulates dendrite patterning. Remarkably, TRPC5 forms a complex specifically with CaMKII $\beta$ but not CaMKII $\alpha$. Accordingly, TRPC5 induces the CaMKII $\beta$-dependent phosphorylation of the E3 ubiquitin ligase Cdc20-APC, and the centrosomal CaMKII $\beta$ signaling pathway mediates TRPC5-dependent dendrite morphogenesis. Together, our findings define a novel function for TRPC5 as a key activator of calcium-regulated ubiquitin signaling at the centrosome, leading to the establishment of neuronal connectivity in the mammalian brain.

\section{Results}

TRPC5 regulates dendrite patterning in primary neurons and in the mammalian brain in vivo

To characterize the role of TRPCs in neuronal development, we used granule neurons of the developing rat cerebellar cortex. Granule neurons provide a robust model system for studies of neuronal morphogenesis and connectivity (Yang et al. 2010; de la Torre-Ubieta and Bonni 2011). Granule neurons are generated in the external granule layer (EGL) in the developing cerebellar cortex. Newly generated granule neurons extend parallel fiber axons and migrate inward through the molecular layer (ML) into the internal granule layer (IGL). Within the IGL, granule neurons elaborate dendrites, which branch extensively, followed by a phase of dendrite retraction and pruning. As they mature, granule neuron dendrites form at their ends specialized structures termed dendritic claws, which house synapses with mossy fiber terminals and Golgi neuron axons (Palay and Chan-Palay 1974; Hamori and Somogyi 1983; Ramon y Cajal 1995; Shalizi et al. 2006). Thus, granule neurons follow a characteristic and stereotyped program of neuronal morphogenesis typical of neurons in the CNS (Hatten and Heintz 1995; Altman and Bayer 1997).

To determine the function of TRPCs in neurons, we employed a plasmid-based method of RNAi to acutely knock down TRPC1, TRPC3, TRPC4, TRPC5, TRPC6, and TRPC7 (Gaudilliere et al. 2002). TRPC family members form homomers or heteromers. TRPC1 forms heteromers with TRPC4 or TRPC5, while TRPC3, TRPC6, and TRPC7 form distinct heteromers (Ramsey et al. 2006). We generated shRNAs that target distinct regions of the TRPCs and confirmed that the shRNAs induced knockdown of each targeted TRPC protein in cells (Supplemental Fig. S1). We next determined the effect of knockdown of each TRPC family member on the morphology of granule neurons dendrites, which are easily identified based on their morphology and immunocytochemical markers (Gaudilliere et al. 2004; Kim et al. 2009; de la Torre-Ubieta et al. 2010). We found that knockdown of members of the TRPC1/4/5 channel subfamily, but not knockdown of the TRPC3/6/7 channel subfamily, led to a robust phenotype characterized by long primary dendrites with increased secondary and tertiary dendrite branching (Fig. 1A). In morphometric analyses, total dendrite length was significantly increased upon TRPC1, TRPC4, or TRPC5 knockdown (Fig. 1B). In contrast, knockdown of TRPC3, TRPC6, and TRPC7 had little or no effect on dendrite length (Fig. 1A,B), suggesting a requirement for members of the TRPC1/4/5 channel subfamily in the restriction of dendrite growth and elaboration in neurons. In other experiments, we found that expression of exogenous TRPC5, but not other TRPCs, was sufficient to restrict dendrite growth (Fig. 1C,D). Together, these data suggest that TRPC5 may play a critical role in the regulation of dendrite morphogenesis.

The finding that TRPC5 is necessary and sufficient to restrict dendrite growth in primary neurons led us to the 
A

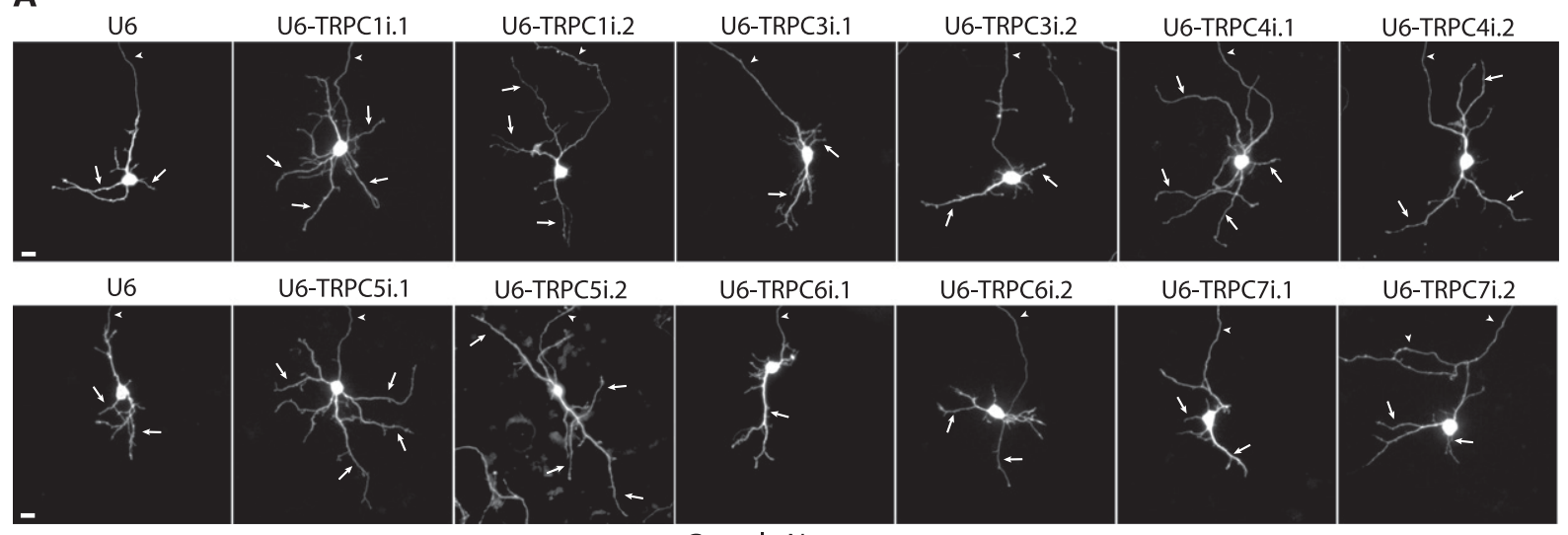

B

Granule Neurons

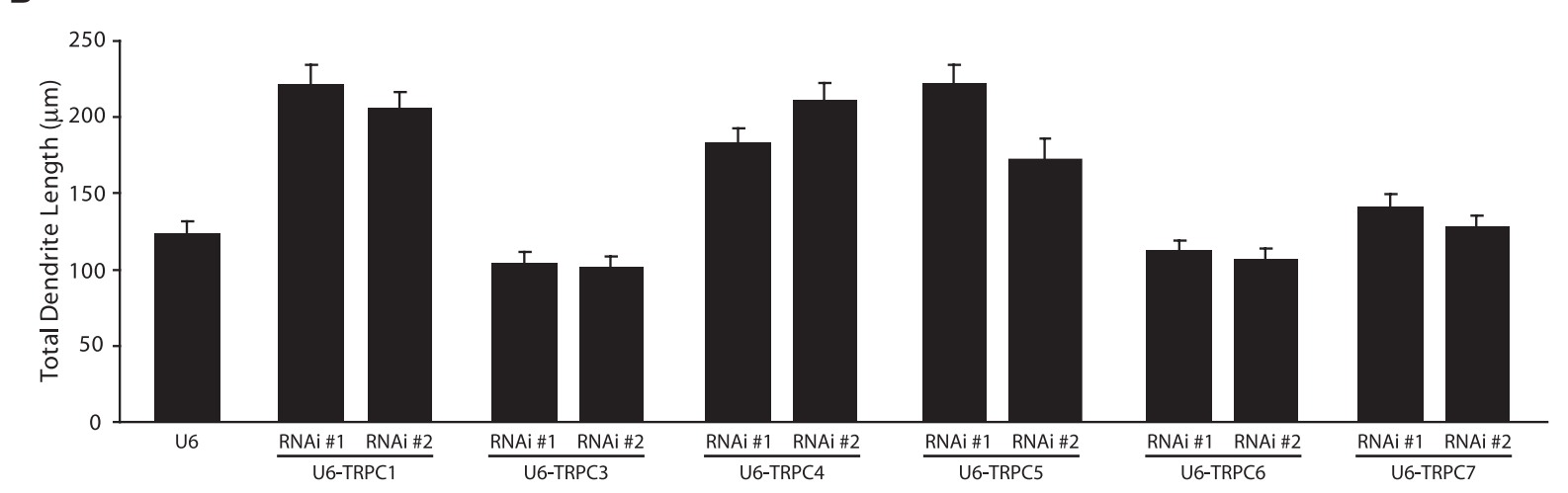

C
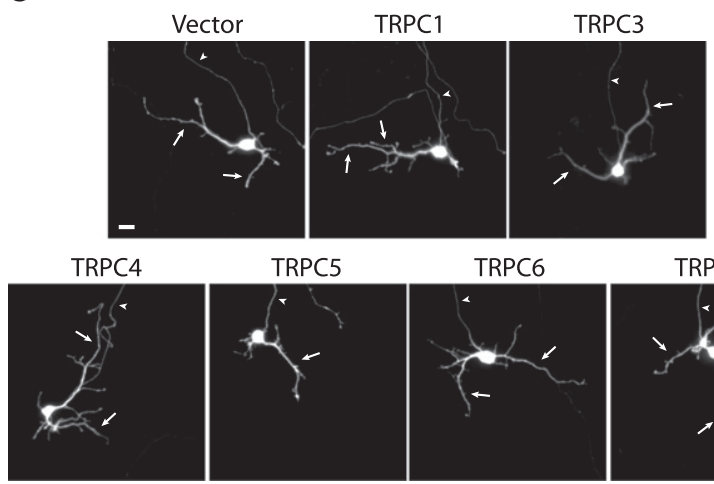

TRPC5 TRPC6 TRPC7
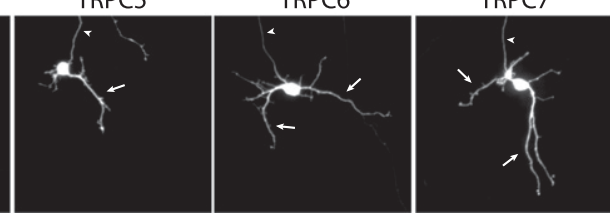

D

Granule Neurons

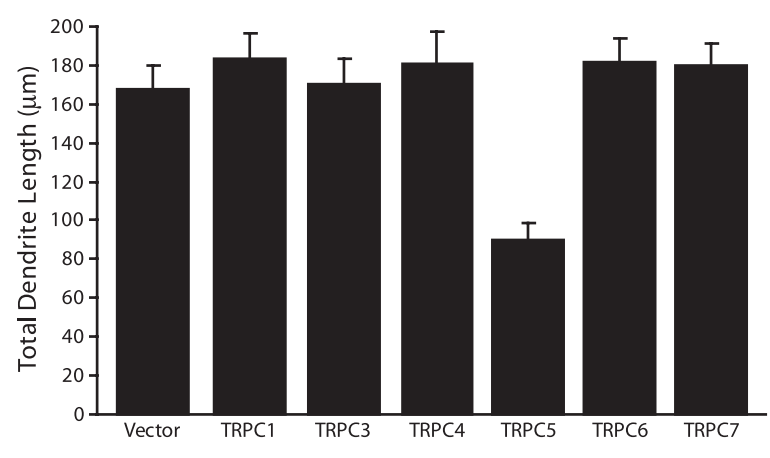

Figure 1. TRPC5 restricts dendrite growth and elaboration in neurons. (A) Granule neurons transfected with distinct TRPC RNAi plasmids targeting TRPC1, TRPC3, TRPC4, TRPC5, TRPC6, TRPC7, or the control U6 plasmid together with an expression plasmid encoding GFP were subjected to immunocytochemistry $4 \mathrm{~d}$ later using the GFP antibody. Representative neurons are shown. In all images of neuronal morphology, arrows and arrowheads indicate dendrites and axons, respectively. TRPC1, TRPC4, and TRPC5 knockdown led to longer, more highly branched dendrites. Bar, $10 \mu \mathrm{m}$. $(B)$ Total dendrite length for granule neurons treated as in $A$ was quantified. Total dendrite length was significantly increased in TRPC1, TRPC4, and TRPC5 knockdown neurons compared with control U6-transfected neurons. In contrast, TRPC3, TRPC6, or TRPC7 knockdown had little or no effect on total dendrite length compared with control U6 transfection (ANOVA, $P<0.0001$ ). One-thousand-one-hundred-seventy neurons were measured. Specific values for mean, SEM, and number of cells analyzed for each condition are provided for all experimental results in Supplemental Table 2. A detailed statistics table comparing each shRNA with the others is provided in Supplemental Table 3. The population distribution of total dendrite length for TRPC5 knockdown and control U6-transfected neurons is shown in Supplemental Figure S10. (C) Granule neurons transfected with an expression plasmid encoding TRPC1, TRPC3, TRPC4, TRPC5, TRPC6, TRPC7, or their control vector together with the GFP expression plasmid were analyzed as in A. Expression of TRPC5-but not TRPC1, TRPC3, TRPC4, TRPC6, or TRPC7-substantially reduced dendrite growth and arborization. Bar, $10 \mu \mathrm{m}$. $(D)$ Total dendrite length for granule neurons treated as in $C$ was quantified. Total dendrite length was significantly decreased in neurons expressing TRPC5-but not in neurons expressing TRPC1, TRPC3, TRPC4, TRPC6, or TRPC7—compared with control vector-transfected neurons (ANOVA, $P<0.0001$ ). Six-hundredthirty neurons were measured. 
question of the function of TRPC5 in dendrite morphogenesis in vivo. Mice in which the TRPC5 gene is disrupted have been recently generated (Riccio et al. 2009), facilitating the analysis of TRPC5 function in dendrite morphogenesis. We first characterized the morphology of primary TRPC5 wild-type and knockout neurons. Cerebellar granule neurons as well as hippocampal neurons from TRPC5 knockout mice displayed more branched dendrites and increased total dendrite length compared with neurons from wild-type littermates (Figs. 2A-C, 3AD). Further characterization revealed that TRPC5 knockout animals had a shift in the distribution of granule neurons toward increased total dendrite length compared with wild-type littermates (Supplemental Fig. S2A). These data corroborate the conclusion that TRPC5 restricts dendrite growth and elaboration in primary mammalian brain neurons.

We next used the TRPC5 knockout mice to determine the role of TRPC5 in dendrite development in the mammalian brain in vivo. We employed a diolistics approach to visualize dendrite arbors in the cerebellar cortex in wild-type and TRPC5 knockout mice. Strikingly, IGL granule neurons in postnatal day 7 (P7) TRPC5 knockout animals had longer dendrites with increased secondary and tertiary dendrite branching than IGL granule neurons in control animals (Fig. 2D). Morphometric analyses revealed a substantial increase in the number of secondary and tertiary dendrite branches and a significant increase in total dendrite length in IGL neurons in TRPC5 knockout animals compared with control animals (Fig. 2F; Supplemental Fig. S2B). These data reveal a physiologic function for TRPC5 in limiting the elaboration of dendrite arbors in the cerebellar cortex in vivo. Similar results were obtained in analyses of pyramidal neurons of the CA1 region of the hippocampus in P7 animals (Fig. $3 \mathrm{E}-\mathrm{H})$. Together, our findings suggest that TRPC5 restricts dendrite growth and elaboration in the mammalian brain in vivo.

To determine whether TRPC5 also regulates dendrite morphogenesis at later stages of development, we analyzed dendrite arbors of IGL granule neurons in P11 TRPC5 wild-type and knockout littermates. In wild-type animals, IGL granule neurons exhibited a few short dendrites with simplified arbors (Fig. 2E,F; Supplemental Fig. S2B), characteristic of the mature stage of dendrite differentiation in granule neurons. In addition, IGL granule neurons harbored dendritic claws (Fig. 2E,G), which house synapses with afferent mossy fiber terminals and Golgi neuron axons (Palay and Chan-Palay 1974; Ramon y Cajal 1995), providing further evidence of dendrite maturation in control P11 animals. In contrast to wild-type littermates, IGL granule neurons in P11 TRPC5 knockout animals displayed longer, more branched dendrite arbors (Fig. 2E,F; Supplemental Fig. S2B), suggesting that disruption of TRPC5 blocks the differentiation of dendrites at the stage of exuberant arbors. Consistent with these observations, IGL granule neuron dendrites in P11 TRPC5 knockout pups had a significantly lower number of dendritic claws (Fig. 2E,G). Just as in the cerebellar cortex, total dendrite length and branching was substantially increased in pyramidal neurons in the CA1 region of the hippocampus in P11 TRPC5 knockout animals compared with wild-type littermates (Fig. 3I-K). Together, these results suggest that TRPC5 plays an essential role in dendrite patterning in the mammalian brain in vivo.

The identification of a physiologic function for TRPC5 in dendrite patterning led us next to the question of whether TRPC5 regulates dendrite development in a cellautonomous manner. To address this question, we took advantage of an in vivo RNAi approach in which gene knockdown is induced in a small percentage of sparsely distributed neurons in the cerebellar cortex in postnatal rat pups in vivo (Konishi et al. 2004; Shalizi et al. 2006; Kim et al. 2009). We first validated the specificity of the shRNAs targeting TRPC5 in primary neurons. We generated an expression plasmid encoding TRPC5 that is resistant to RNAi (TRPC5-RES) (Fig. 4A). Expression of TRPC5-RES, but not TRPC5 encoded by wild-type cDNA (TRPC5-WT), restored the typical appearance of dendrite arbors and reduced dendrite length and branching in the background of TRPC5 RNAi to that of control transfected neurons (Fig. 4B,C; Supplemental Fig. S3A-C). In other experiments, knockdown of TRPC5 in wild-type mouse granule neurons led to a dendrite phenotype similar to that of TRPC5 knockout granule neurons, and knockdown of TRPC5 had little or no additive effect on the dendrite phenotype in TRPC5 knockout neurons (Supplemental Fig. S3D), further strengthening the conclusion that TRPC5 RNAi does not impair dendrite development via off targets of TRPC5 shRNAs or nonspecific activation of the RNAi machinery. In other control analyses, expression of TRPC5 in granule neurons from TRPC5 knockout mice suppressed the dendrite phenotype in these neurons (Supplemental Fig. S3E).

Having validated the specificity of the TRPC5 shRNAs, we next determined whether TRPC5 regulates dendrite morphogenesis in a cell-autonomous manner in vivo. We electroporated P3 rat pups with a TRPC5 RNAi plasmid that coexpresses GFP (U6-TRPC5i/CMV-GFP) or the corresponding control RNAi plasmid (U6/CMV-GFP) (Fig. 4D-H). Five days or $9 \mathrm{~d}$ after electroporation, animals were sacrificed and cerebella were subjected to immunohistochemical analysis using the GFP antibody (Fig. 4D$\mathrm{H})$. Analyses of the cerebellar cortex in P8 and P12 rat pups revealed that granule neuron dendrites were longer and more elaborate in TRPC5 knockdown animals compared with control animals (Fig. 4D-G; Supplemental Fig. S3F,G). In addition, IGL granule neurons in P12 TRPC5 knockdown rats harbored fewer dendritic claws compared with control animals (Fig. 4H). These data suggest that TRPC5 restricts the elaboration of dendrite arbors and promotes their maturation in a cell-autonomous manner in vivo. Taken together, our findings suggest that TRPC5 acts as a critical, cell-autonomous regulator of dendrite morphogenesis during development.

To further determine the role of TRPC5 in dendrite patterning, we induced knockdown of TRPC5 in granule neurons in the cerebellar cortex specifically at the stage of exuberant dendrites at a time when neurons have already elaborated dendrite arbors and begin to undergo dendrite 
A

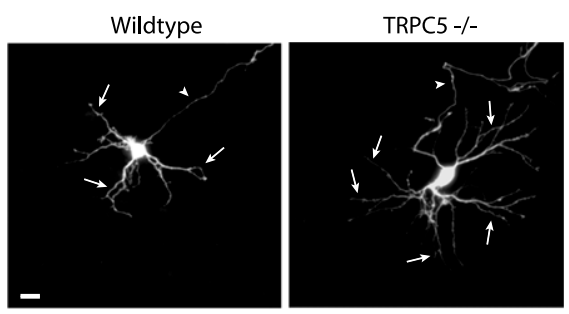

Granule Neurons
B

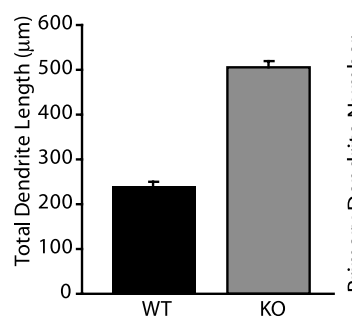

C

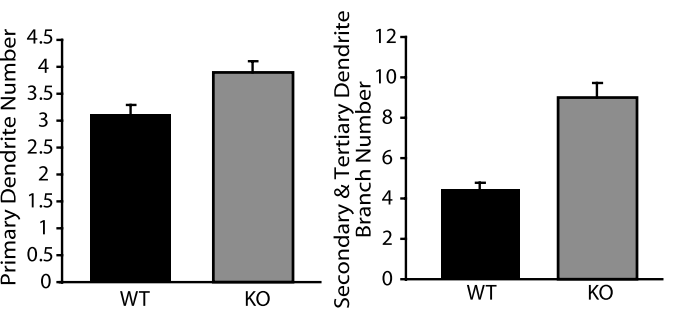

D

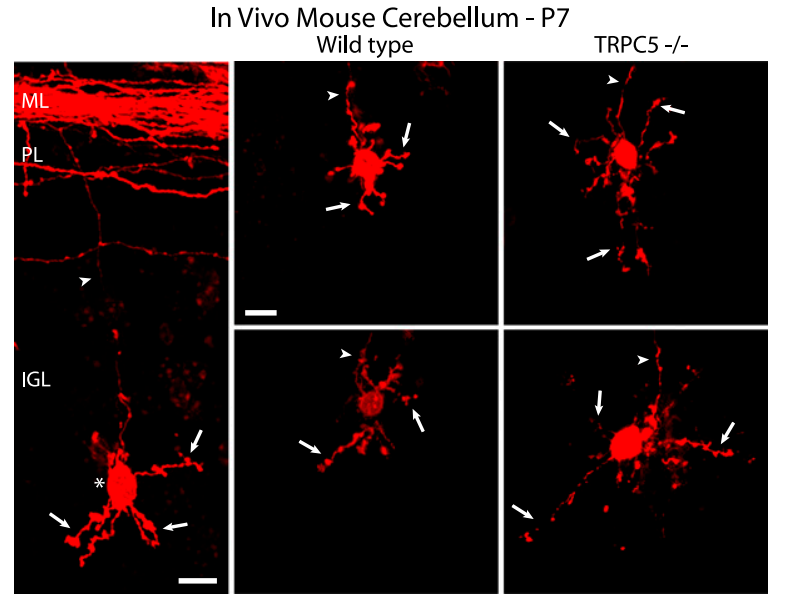

E

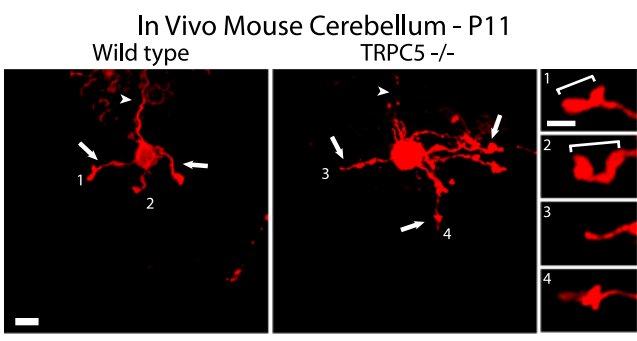

$\mathbf{F}$

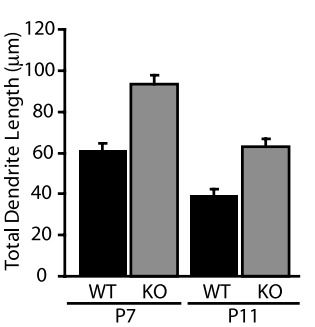

G

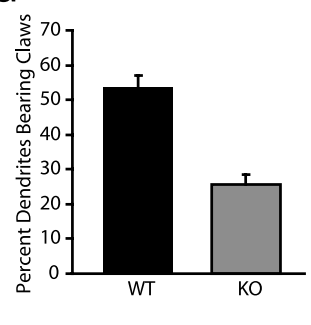

Figure 2. TRPC5 is essential for dendrite patterning in the mammalian brain. (A) Granule neurons from TRPC5 wild-type and knockout littermates transfected with GFP were analyzed as in Figure 1A. Representative neurons are shown. Granule neurons from TRPC5 knockout mice had longer, more highly branched dendrites compared with neurons from wild-type littermates. Bar, $10 \mu \mathrm{m}$. $(B)$ Granule neurons from TRPC5 wild-type and knockout littermates were treated as in $A$ and subjected to morphometric analysis. Granule neurons from TRPC5 knockout animals had significantly increased total dendrite length compared with neurons from wildtype littermates $(t$-test, $P<0.0001)$. One-hundred-sixty-four neurons were measured in six animals (three wild type and three TRPC5 knockout). (C) Granule neurons from TRPC5 wild-type and knockout littermates were treated as in $A$ and subjected to morphometric analysis. Primary dendrite number was modestly increased $(t$-test, $P<0.005)$, and secondary and tertiary dendrite branch number was significantly increased in granule neurons from TRPC5 knockout mice compared with wild-type littermates $(t$-test, $P<0.001)$. Onehundred-sixty-four neurons were analyzed in six animals (three wild type and three TRPC5 knockout). (D) P7 wild-type and TRPC5 knockout littermate mice were sacrificed, and cerebella were subjected to analysis using a diolistics approach. (Left) Representative wild-type granule neuron, with soma (asterisk) and dendrites (arrows) in the IGL and ascending axon (arrowhead) connecting to the horizontally oriented parallel fibers superficially. Bar, $10 \mu \mathrm{m}$. (Right) Representative IGL granule neurons in wild-type and TRPC5 knockout animals are shown. IGL granule neurons in TRPC5 knockout animals had longer, more highly branched dendrites compared with IGL granule neurons in wild-type littermates. Bar, $10 \mu \mathrm{m}$. (E) P11 wild-type and TRPC5 knockout littermate mice were sacrificed, and cerebella were analyzed as in $D$. Representative IGL granule neurons in wild-type and TRPC5 knockout animals are shown. Bar, 10 $\mu \mathrm{m}$. (Inset) Zoomed view of dendritic tips of individual neurons. Bar, $2.5 \mu \mathrm{m}$. Bracket identifies dendritic claws. IGL granule neurons in TRPC5 knockout animals had longer, more highly branched dendrites with fewer dendritic claws compared with IGL granule neurons in wild-type littermates. (F) IGL granule neurons analyzed as in $D$ and $E$ were subjected to morphometric analysis. Total dendrite length was significantly increased in IGL granule neurons in P7 and P11 TRPC5 knockout animals compared with wild-type littermates (ANOVA, $P<0.0001$ ). Three-hundred-seventy-eight neurons were measured in 12 animals (three wild type and three TRPC5 knockout for each age). $(G)$ IGL granule neurons analyzed as in $E$ were subjected to morphometric analysis. The percentage of dendrites bearing claws was significantly reduced in IGL granule neurons in P11 TRPC5 knockout animals compared with wild-type littermates $(t$-test, $P<0.0001$ ). One-hundred-eighty neurons were analyzed in six animals (three wild type and three TRPC5 knockout).

retraction and pruning. We therefore induced TRPC5 knockdown in cerebellar slices prepared from P10 rat pups, using a biolistic approach. This method allows acute knockdown of genes in a small percentage of neurons within the relatively intact architecture of the cerebellar cortex, including the IGL /Gaudilliere et al.
2004; Kim et al. 2009). We found that knockdown of TRPC5 in P10 cerebellar slices at the stage of exuberant dendrites impaired subsequent dendrite pruning and retraction (Supplemental Fig. S3H). Accordingly, IGL granule neurons in TRPC5 knockdown cerebellar slices had substantially increased total dendrite length and fewer 
A

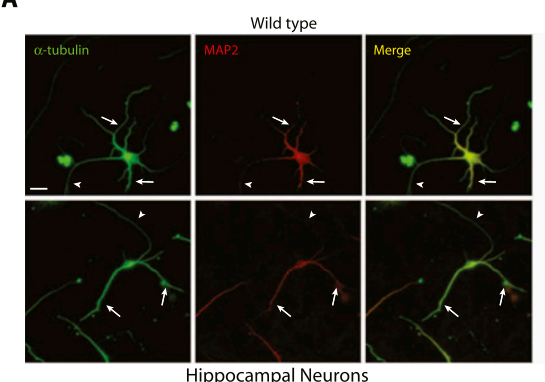

Hippocampal Neurons

B

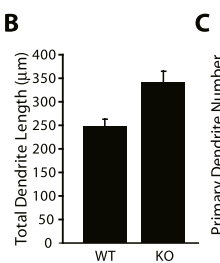

c
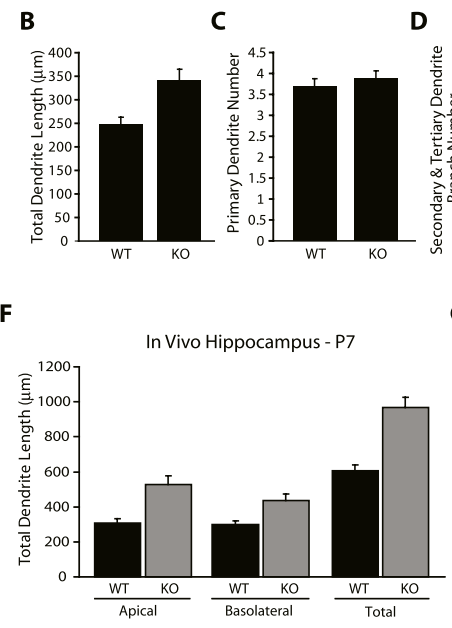

I

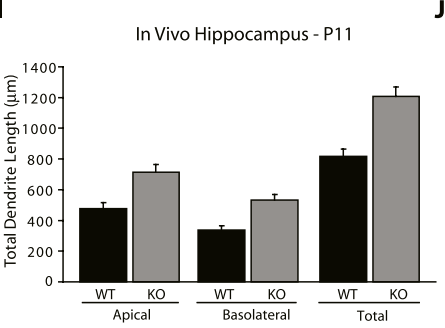

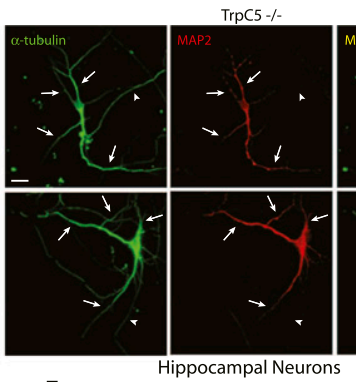

E

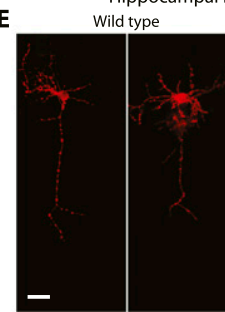

P7 CA1 Pyramidal Neurons
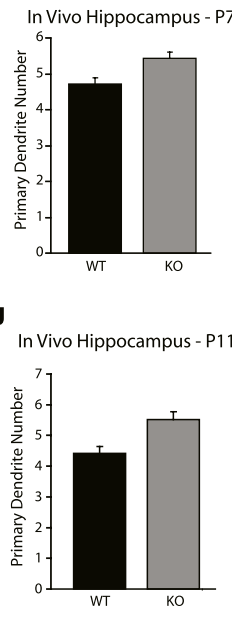

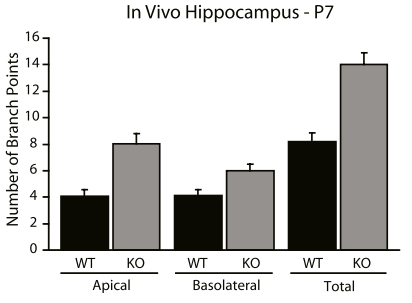

K

In Vivo Hippocampus - P11

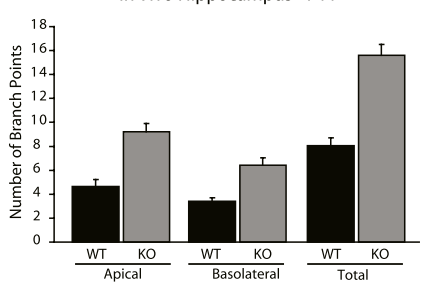

Figure 3. TRPC5 knockout stimulates dendrite growth and arborization in hippocampal neurons. (A) Hippocampal neurons from TRPC5 wild-type and knockout littermates were subjected to immunocytochemistry using the $\alpha$-tubulin and MAP2 antibody. Representative neurons are shown. TRPC5 knockout neurons had longer, more branched dendrites as compared with neurons from wild-type littermates. Bar, $20 \mu \mathrm{m}$. (B) Hippocampal neurons from TRPC5 wild-type and knockout littermates were analyzed as in $A$ and total dendrite length was quantified. Total dendrite length was significantly increased in TRPC5 knockout neurons compared with neurons from wild-type littermates ( $t$-test, $P<0.005$ ). One-hundred neurons were measured in six animals (three wild type and three TRPC5 knockout). (C) Hippocampal neurons from TRPC5 wild-type and knockout littermates were analyzed as in $A$ and primary dendrite number was quantified. Primary dendrite number was not significantly different in TRPC5 knockout neurons compared with neurons from wildtype littermates. One-hundred neurons were analyzed in six animals (three wild type and three TRPC5 knockout). (D) Hippocampal neurons from TRPC5 wild-type and knockout littermates were analyzed as in $A$ and secondary and tertiary dendrite branch number was quantified. Secondary and tertiary dendrite branch number was significantly increased in TRPC5 knockout neurons compared with neurons from wildtype littermates (t-test, $P<0.01)$. Onehundred neurons were analyzed in six animals (three wild type and three TRPC5 knockout). (E) P7 wild-type and TRPC5 knockout animals were sacrificed and hippocampal sections were analyzed using a diolistics approach as in Figure 2D. Representative CA1 pyramidal neurons in wild-type and TRPC5 knockout animals are shown. CA1 pyramidal neurons in TRPC5 knockout animals had longer, more highly branched dendrites compared with CA1 pyramidal neurons in wild-type littermates. Bar, $25 \mu \mathrm{m}$. $(F)$ CA1 pyramidal neurons analyzed as in $E$ were subjected to morphometric analysis. Basolateral dendrite length was modestly increased, and apical and total dendrite length were significantly increased in CA1 pyramidal neurons in TRPC5 knockout animals compared with wild-type littermates $($ ANOVA, $P<$ 0.0001). Ninety neurons were measured in six animals (three wild type and three TRPC5 knockout). (G) CA1 pyramidal neurons analyzed as in $E$ were subjected to morphometric analysis. Primary dendrite number was modestly increased in CA1 pyramidal neurons in TRPC5 knockout animals compared with wild-type littermates ( $t$-test, $P<0.01$ ). Ninety neurons were measured in six animals (three wild type and three TRPC5 knockout). $(H)$ CA1 pyramidal neurons analyzed as in $E$ were subjected to morphometric analysis. The number of basolateral dendrite branch points was modestly increased, and apical and total dendrite branch points were significantly increased in CA1 pyramidal neurons in TRPC5 knockout animals compared with wild-type littermates (ANOVA, $P<0.0001)$. Ninety neurons were analyzed in six animals (three wild type and three TRPC5 knockout). (I) P11 wild-type and TRPC5 knockout animals were sacrificed, and hippocampal sections were analyzed using a diolistics approach as in $E$ and subjected to morphometric analysis. Basolateral dendrite length was modestly increased, and apical and total dendrite length were significantly increased in CA1 pyramidal neurons in TRPC5 knockout animals compared with wild-type littermates (ANOVA, $P<0.0001)$. Ninety neurons were measured in six animals (three wild type and three TRPC5 knockout). (J) CA1 pyramidal neurons analyzed as in $I$ were subjected to morphometric analysis. Primary dendrite number was modestly increased in CA1 pyramidal neurons in TRPC5 knockout animals compared with wild-type littermates ( $t$-test, $P<0.005)$. Ninety neurons were measured in six animals (three wild type and three TRPC5 knockout). $(K)$ CA1 pyramidal neurons analyzed as in $I$ were subjected to morphometric analysis. The number of basolateral dendrite branch points was modestly increased, and apical and total dendrite branch points were significantly increased in CA1 pyramidal neurons in TRPC5 knockout animals compared with wild-type littermates (ANOVA, $P<0.0001$ ). Ninety neurons were analyzed six animals (three wild type and three TRPC5 knockout). 
A

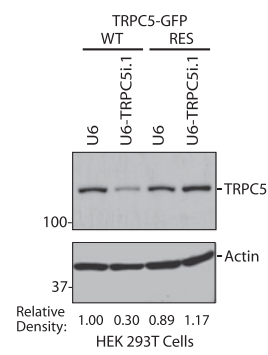

D

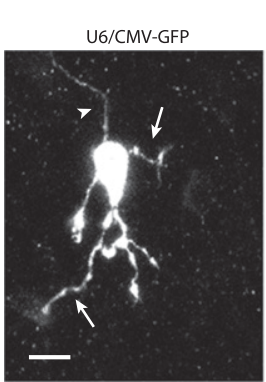

$\mathbf{F}$

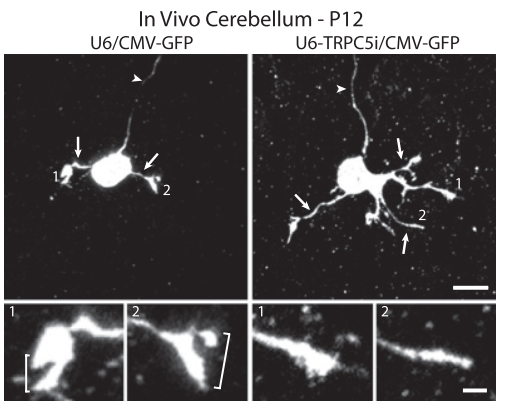

B

In Vivo Cerebellum - P8

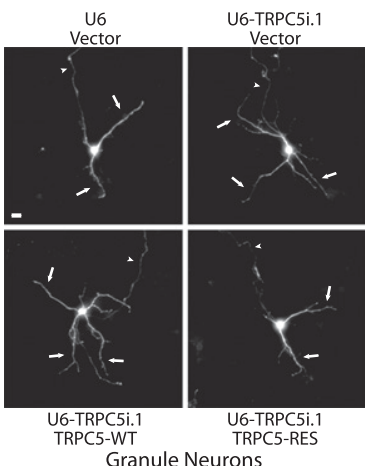

Granule Neurons

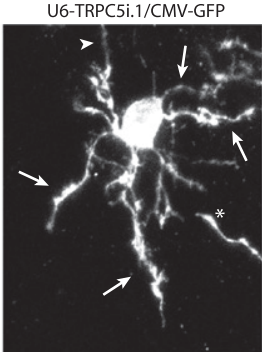

U6-TRPC5i.2/CMV-GFP

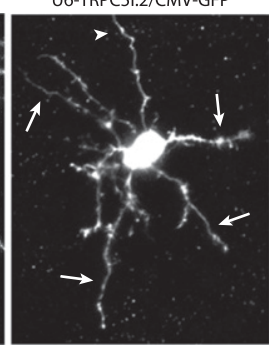

G

In Vivo Cerebellum - P12

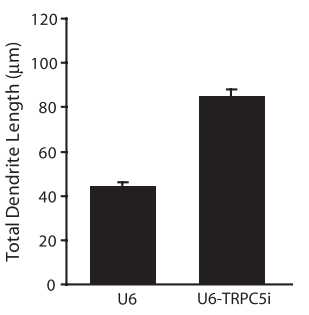

C

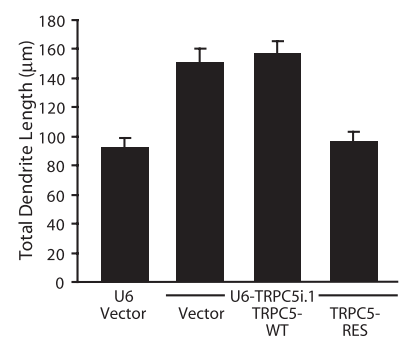

E

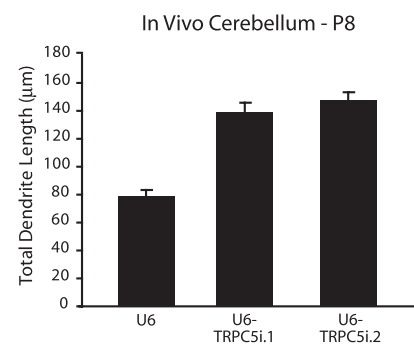

H

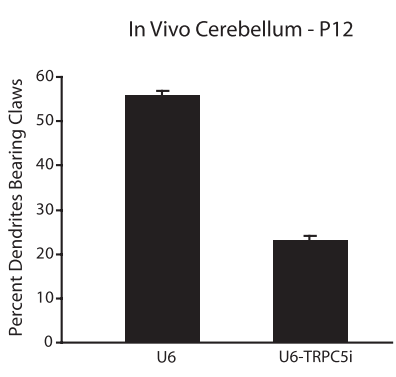

Figure 4. TRPC5 drives dendrite patterning in a cell-autonomous manner in vivo. (A) Lysates of $293 \mathrm{~T}$ cells transfected with an expression plasmid encoding TRPC5-WT-GFP or TRPC5-RES-GFP together with the TRPC5 RNAi or control U6 plasmid were immunoblotted with the GFP or Actin antibody. The relative density of the TRPC5-GFP band (normalized to Actin) is shown below each lane. $(B)$ Granule neurons transfected with the TRPC5 RNAi or control U6 plasmid together with the expression plasmid encoding TRPC5-WT, TRPC5-RES, or control vector and the GFP expression plasmid were analyzed as in Figure 1A. Expression of TRPC5-RES, but not TRPC5-WT, substantially reduced dendrite growth and arborization compared with control vector in the background of TRPC5 RNAi. Bar, $10 \mu \mathrm{m} .(C)$ Total dendrite length for granule neurons treated as in $B$ was quantified. Expression of TRPC5-RES, but not TRPC5WT, significantly reduced total dendrite length compared with control vector in the background of TRPC5 RNAi (ANOVA, $P<0.0001$ ). Three-hundred-sixty neurons were measured. $(D)$ Rat pups electroporated in vivo with a U6-TRPC5i/CMV-GFP RNAi or control U6/ CMV-GFP plasmid were sacrificed $5 \mathrm{~d}$ after electroporation (P8), and cerebella $(\mathrm{Cb})$ were subjected to immunohistochemistry using the GFP and Calbindin antibody. Representative neurons for each condition are shown. IGL granule neurons in TRPC5 knockdown animals had longer, more highly branched dendrites than IGL granule neurons in control U6 animals. The asterisk indicates process from another neuron. Bar, $10 \mu \mathrm{m}$. (E) IGL granule neurons analyzed as in $D$ were subjected to morphometric analysis. Total dendrite length was significantly increased in IGL granule neurons in TRPC5 knockdown animals compared with control U6 animals (ANOVA, $P<0.0001$ ). Two-hundred-forty-six neurons were measured in nine animals (three for each condition). (F) Rat pups electroporated in vivo with the U6TRPC5i/CMV-GFP RNAi or control U6/CMV-GFP plasmid were sacrificed at P12 and analyzed as in D. Representative IGL granule neurons for each condition are shown. Bar, $10 \mu \mathrm{m}$. (Inset) Zoomed view of dendritic tips of individual neurons. Bar, $2.5 \mu \mathrm{m}$. Bracket identifies dendritic claws. IGL granule neurons in TRPC5 knockdown animals had longer, more highly branched dendrites with fewer dendritic claws than IGL granule neurons in control U6 animals. $(G)$ IGL granule neurons analyzed as in $F$ were subjected to morphometric analysis. Total dendrite length was significantly increased in IGL granule neurons in TRPC5 knockdown animals compared with control U6 animals ( $t$-test, $P<0.0001)$. One-hundred-eighty-four neurons were measured in six animals (three control and three knockdown). (H) IGL granule neurons analyzed as in $F$ were subjected to morphometric analysis. The percentage of dendrites bearing claws was significantly decreased in IGL granule neurons in TRPC5 knockdown animals compared with control U6 animals ( $t$-test, $P<0.0001)$. One-hundred-eighty-four neurons were analyzed in six animals (three control and three knockdown). 
dendritic claws than granule neurons in control cerebellar slices (Supplemental Fig. S3I,J), suggesting that TRPC5 knockdown neurons arrest at the developmental stage of exuberant dendrites prior to their pruning. Collectively, these data suggest that TRPC5 plays a critical role in driving dendrite patterning in the cerebellar cortex.

\section{TRPC5 knockout mice have motor coordination deficits}

The identification of a critical role for TRPC5 in dendrite morphogenesis in the cerebellar cortex led us to ask whether TRPC5 knockout mice have deficits in motor function. We first characterized TRPC5 wild-type and knockout littermates using balance beam assays (Fig. 5A). In analyses using a wide $(20 \mathrm{~mm}$ wide) balance beam, wild-type and TRPC5 knockout littermates performed comparably (data not shown). However, when challenged with a narrow $(4 \mathrm{~mm}$ wide) balance beam, TRPC5 knockouts had over twofold more foot slips than wildtype littermates (Fig. 5B; Supplemental Movie S1). There was no difference in mean crossing time between knockout and wild-type littermates (Fig. 5C; Supplemental Movie S1), suggesting that foot-slip errors in TRPC5 knockout animals were not due to differences in walking speed compared with wild-type animals.

To further characterize motor coordination in TRPC5 knockout mice, we examined gait parameters in wildtype and knockout littermates using a treadmill equipped with a ventral plane, high-frame-rate video imaging system (Digigait) (Supplemental Fig. S4A; Supplemental Movie S2). Analyses of digital paw prints captured during ambulation provided measurements of numerous gait parameters (Hurlock et al. 2009; Kravitz et al. 2010). In these analyses, stride length was reduced while stride frequency was increased in TRPC5 knockout mice compared with wild-type littermates (Supplemental Table 1). In addition, TRPC5 knockout mice had increased step-tostep variability in stride length and paw angle (Supplemental Table 1), consistent with an ataxic gait (Palliyath et al. 1998; Ebersbach et al. 1999). Correspondingly, the ataxia coefficient, which measures deviation of the minimum and maximum stride length from the mean, was higher in TRPC5 knockouts (Supplemental Table 1). Importantly, the relative duration of each phase of gait was unaltered between the two groups (Supplemental Table 1), suggesting that individual limbs still moved normally through the distinct phases. Analyses of grip strength, metabolic activity, forced swim, Y maze spontaneous alternation, and contextual fear conditioning revealed little or no difference in the performance of TRPC5 knockout animals compared with wild-type littermates (Supplemental Fig. S5). In addition, TRPC5 knockout mice did not have deficits in spontaneous behavior, neurological reflexes, or sensorimotor responses-including righting, postural reflex, ear twitch reflex, and whisker orientationcompared with wild-type mice (Riccio et al. 2009). Taken together, our results suggest that TRPC5 plays a critical and specific role in motor coordination.
We next characterized dendrite morphology in the cerebellar cortex of adult wild-type and TRPC5 knockout littermates with the aim of determining whether impaired dendrite morphology correlates with impaired motor coordination. Just as in the developing cerebellar cortex, we found that granule neurons in adult TRPC5 knockout mice had long, highly branched dendrites with fewer dendritic claws compared with granule neurons from wild-type littermates (Fig. 5D-G). Notably, within the group of TRPC5 knockout mice, both the increased total dendrite length and impaired dendritic claw formation in the cerebellar cortex of individual mice correlated with a greater number of foot-slip errors (Fig. 5H,I). A similar relationship was observed between the ataxia coefficient and increased total dendrite length or impaired dendritic claw formation in TRPC5 knockout mice (Supplemental Fig. S4B,C). These data reveal that the impairment of dendrite morphology in the cerebellar cortex in TRPC5 knockout mice persists into adulthood and correlates with behavioral deficits in motor coordination.

\section{TRPC5 activates centrosomal CaMKII $\beta$ signaling and thereby regulates dendrite patterning}

The identification of a novel function for TRPC5 in the regulation of dendrite patterning and connectivity raised the fundamental question of the molecular basis of TRPC5 function in neurons. As a channel that allows calcium entry in neurons, we reasoned that TRPC5 might regulate the activity of a calcium-responsive signaling protein. We recently found that the major protein kinase CaMKII $\beta$ phosphorylates the E3 ubiquitin ligase Cdc20$\mathrm{APC}$ at the centrosome and thereby triggers dendrite retraction and pruning (Puram et al. 2011). Knockdown of CaMKII $\beta$ closely phenocopies the effect of TRPC5 knockdown or knockout on dendrite morphogenesis, raising the exciting hypothesis that TRPC5 might regulate centrosomal CaMKII $\beta$ signaling in neurons. To investigate this possibility, we first asked whether TRPC5 regulation of dendrite morphogenesis depends on calcium influx. We found that expression of a dominant interfering form of TRPC5 (TRPC5-DN), which blocks whole-cell currents (Greka et al. 2003), markedly stimulated dendrite arbor elaboration, leading to increased dendrite length (Supplemental Fig. S6A,B). In a complementary pharmacological approach, activation of TRPC5 with lanthanum chloride reduced dendrite length, whereas TRPC5 inhibition with SKF96365 or flufenamic acid increased dendrite length (Supplemental Fig. S6C,D). Together, these results suggest that TRPC5-mediated influx of calcium may contribute to TRPC5 restriction of dendrite arbors.

TRPC5 and CaMKII $\beta$ are both expressed at the time of dendrite development in the cerebellar cortex (Fig. 6A), consistent with the possibility that these two proteins might function in a shared pathway to control dendrite morphogenesis. To investigate a potential link between TRPC5 and CaMKII $\beta$, we assessed whether TRPC5 and CaMKII $\beta$ interact in neurons. We found that endogenous CaMKII $\beta$ coprecipitated with TRPC5 immunoprecipitates in wild-type but not TRPC5 knockout lysates (Fig. 

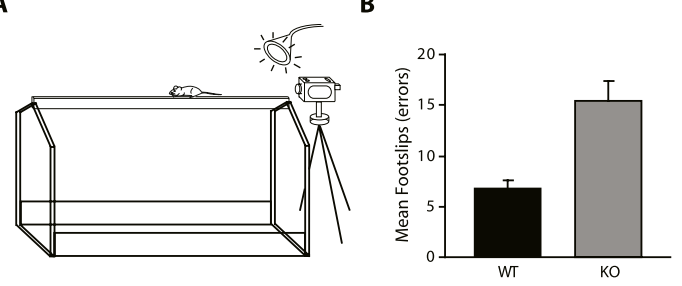

D

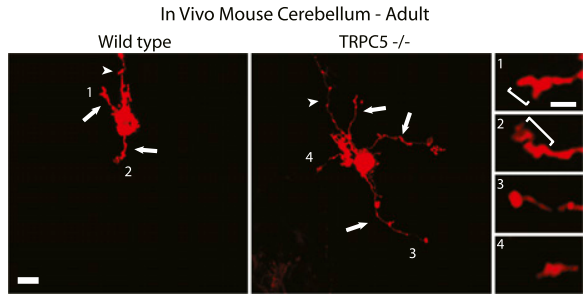

$\mathbf{F}$
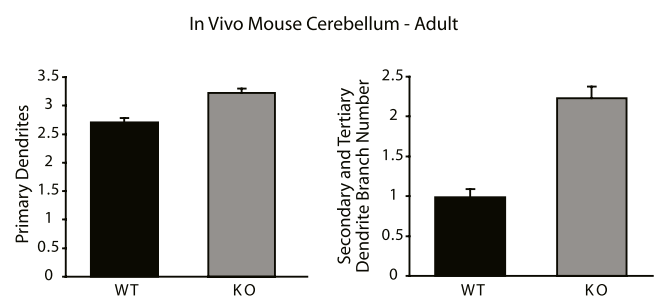

I

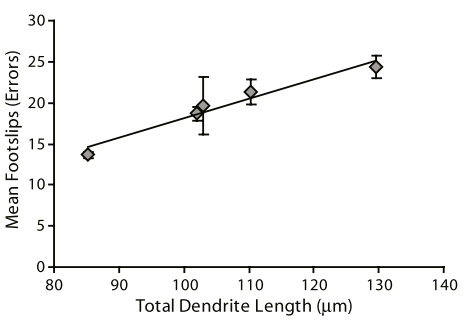

C

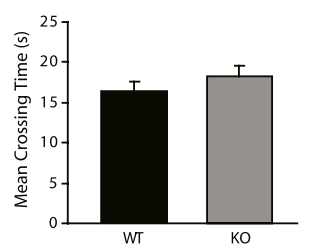

E In Vivo Mouse Cerebellum - Adult

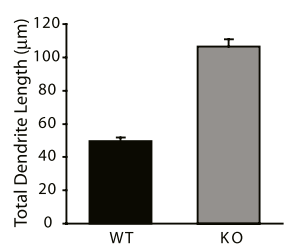

G
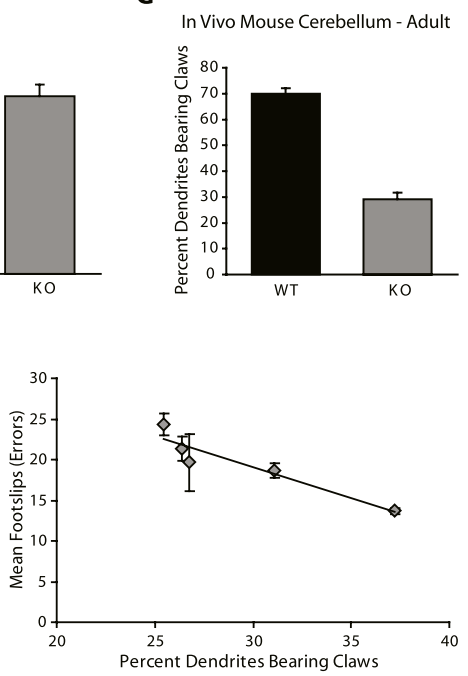

Figure 5. TRPC5 knockout mice have motor coordination deficits. (A) Schematic of balance beam assay used to assess motor coordination of wild-type and TRPC5 knockout littermates. $(B)$ Number of foot slips (errors) on a narrow (4-mmwide) balance beam was quantified in wild-type and TRPC5 knockout littermates. Knockout mice had significantly more foot slips than wild-type littermates $(t$-test, $P<0.01)$. Sixteen littermates were analyzed (eight wild type and eight TRPC5 knockout). (C) Mean crossing time in wild-type and TRPC5 knockout littermates analyzed as in $B$ was quantified. Wild-type and TRPC5 knockout mice did not have significantly different mean crossing times. Sixteen littermates were analyzed (eight wild type and eight TRPC5 knockout). (D) Adult wild-type and TRPC5 knockout littermate mice were sacrificed, and cerebella were analyzed as in Figure 2D. Representative IGL granule neurons in wild-type and TRPC5 knockout animals are shown. Bar, $10 \mu \mathrm{m}$. (Inset) Zoomed view of dendritic tips of individual neurons. Bar, $2.5 \mu \mathrm{m}$. The bracket identifies dendritic claws. IGL granule neurons in adult TRPC5 knockout animals had longer, more highly branched dendrites with fewer dendritic claws compared with IGL granule neurons in wild-type littermates. $(E)$ IGL granule neurons analyzed as in $D$ were subjected to morphometric analysis. Total dendrite length was significantly increased in IGL granule neurons in adult TRPC5 knockout animals compared with wild-type littermates ( $t$-test, $P<0.0001)$. Twohundred-ninety-one neurons were measured in 10 animals (five wild type and five TRPC5 knockout). (F) IGL granule neurons analyzed as in $D$ were subjected to morphometric analysis. Primary dendrite number was modestly increased ( $t$-test, $P<0.0001)$, and secondary and tertiary dendrite branch number was significantly increased in adult TRPC5 knockout animals compared with wild-type littermates ( $t$-test, $P<0.0001)$. Twohundred-ninety-one neurons were analyzed in 10 animals (five wild type and five TRPC5 knockout). (G) IGL granule neurons analyzed as in $D$ were subjected to morphometric analysis. The percentage of dendrites bearing claws was significantly reduced in IGL granule neurons in adult TRPC5 knockout animals compared with wild-type littermates $(t$-test, $P<0.0001)$. Two-hundred-ninety-one neurons were analyzed in 10 animals (five wild type and five TRPC5 knockout). (H) Adult TRPC5 knockout animals tested in behavioral assays were sacrificed, and cerebella were analyzed as in $D$. Total dendrite length and the number of mean foot slips (errors) were plotted for each individual animal. There was a statistically significant correlation between increased total dendrite length and a greater number of foot slips (errors) (Pearson's correlation coefficient 0.953, $P<0.005)$. One-hundred-forty-eight neurons were measured in five animals. (I) Adult TRPC5 knockout animals tested in behavioral assays were analyzed as in $H$. The percentage of dendrites bearing claws and the number of mean foot slips (errors) were plotted for each individual animal. There was a statistically significant correlation between decreased percentage of dendrites bearing claws and a greater number of foot slips (errors) (Pearson's correlation coefficient $0.885, P<$ 0.01). One-hundred-forty-eight neurons were measured in five animals.

$6 \mathrm{~B})$, suggesting that TRPC5 and CaMKII $\beta$ form a complex in neurons. In complementary analyses, endogenous TRPC5 coprecipitated with CaMKII $\beta$ immunoprecipitates (Supplemental Fig. S7A,B). We next determined whether this interaction was specific to the $\beta$ isoform of CaMKII, which drives dendrite retraction and pruning (Puram et al. 2011), but not the $\alpha$ isoform, which promotes dendrite growth and elaboration (Gaudilliere et al. 2004). Remarkably, we found that endogenous TRPC5 interacted specifically with endogenous CaMKII $\beta$, but not CaMKII $\alpha$, in neurons (Fig. 6C). Consistent with these findings, structure-function analyses revealed that the C-terminal portion of the variable region, a domain found in CaMKII $\beta$ but not CaMKII $\alpha$, was required for the interaction with TRPC5 (Supplemental Fig. S7C). In corollary analyses, the cytosolic N-terminal domain of TRPC5 was sufficient for its interaction with CaMKII $\beta$ (Fig. 6D). In contrast to TRPC5, other TRPC family members failed to form a complex with CaMKII (Supplemental Fig. S7D). Together, these results suggest that TRPC5 forms a specific complex with CaMKII $\beta$ and might therefore directly regulate CaMKII $\beta$ function in neurons. 
A

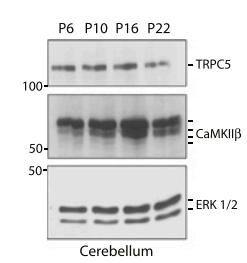

D

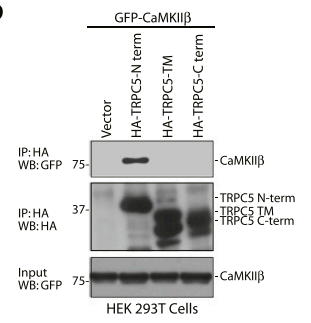

G

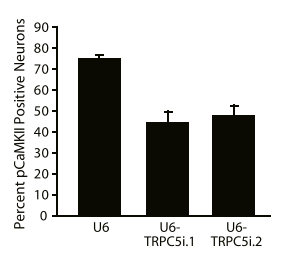

J

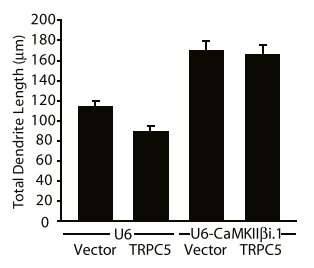

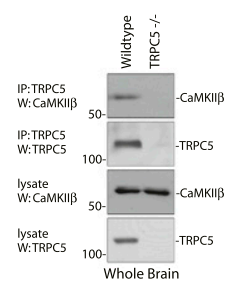

E

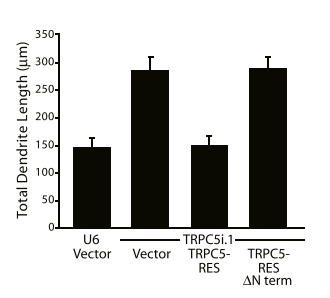

H

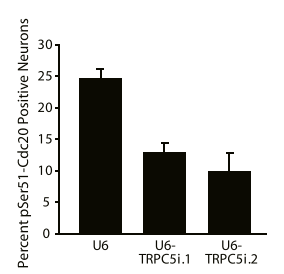

K

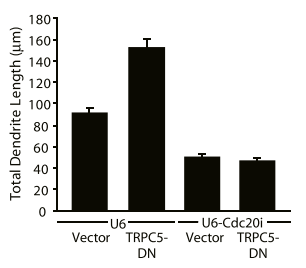

C

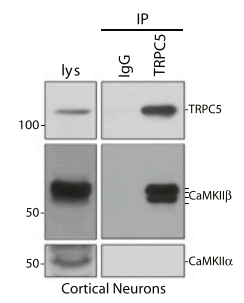

$\mathbf{F}$

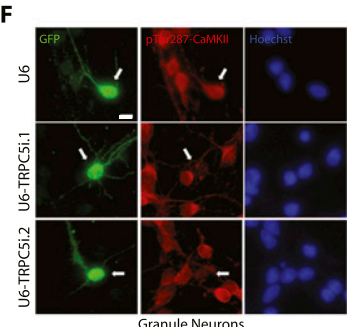

I

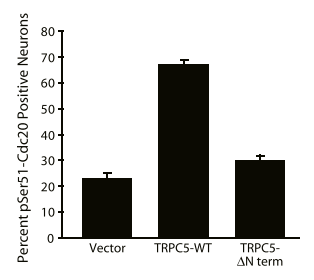

L

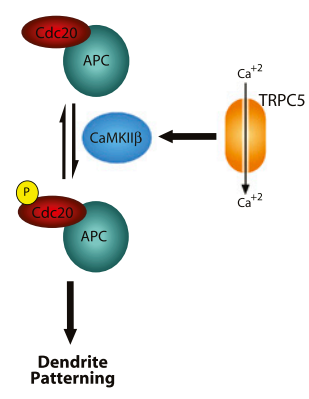

Figure 6. TRPC5 promotes centrosomal CaMKII signaling and thereby regulates dendrite morphogenesis. (A) Whole-cerebellar lysates were immunoblotted with the TRPC5, CaMKII $\beta$, or ERK1/2 antibody. (B) Whole-brain lysates from wild-type and TRPC5 knockout littermates were immunoprecipitated with TRPC5-conjugated agarose beads and immunoblotted with the CaMKII $\beta$ or TRPC5 antibody. Endogenous CaMKII $\beta$ formed a complex with endogenous TRPC5 in wild-type but not TRPC5 knockout neurons. $(C)$ Lysates of cortical neurons were immunoprecipitated with TRPC5- or IgG-conjugated agarose beads and immunoblotted with the CaMKII $\beta$, CaMKIII $\alpha$, or TRPC5 antibody. Endogenous CaMKII $\beta$, but not CaMKII $\alpha$, formed a complex with endogenous TRPC5 in neurons. (D) Lysates of 293T cells transfected with the GFP-CaMKII $\beta$ expression plasmid together with an expression plasmid encoding the $\mathrm{N}$ terminus (HA-TRPC5- $\mathrm{N}$ term), transmembrane domain (HA-TRPC-TM), or C terminus (HATRPC5-C term) of TRPC5, or control vector were immunoprecipitated using the HA antibody and immunoblotted with the GFP or HA antibody. $(E)$ Granule neurons transfected with the TRPC5 RNAi or control U6 plasmid together with the expression plasmid encoding TRPC5-RES, TRPC5RES $\Delta \mathrm{N}$ term, or control vector and the GFP expression plasmid were analyzed as in Figure 1A. TRPC5-RES, but not TRPC5-RES $\Delta \mathrm{N}$ term, significantly reduced granule neuron dendrite length compared with control vector in the background of TRPC5 RNAi (ANOVA, $P<0.0001$ ). Two-hundred-forty neurons were measured. $(F)$ Granule neurons transfected with of one of two different TRPC5 RNAi plasmids (U6-TRPC5i) or the control U6 plasmid together with the GFP expression plasmid were subjected to immunocytochemistry using the GFP or phosphoThr287CaMKII antibody. Arrows indicate transfected neurons. TRPC5 knockdown substantially reduced the phosphorylation of CaMKII $\beta$ at Thr287 in neurons. Bar, $10 \mu \mathrm{m}$. (G) Granule neurons treated as in $F$ were quantified for phosphoThr287-CaMKII $\beta$ signal. The percentage of neurons with phosphoThr287-CaMKII immunoreactivity was significantly reduced in TRPC5 knockdown neurons compared with control U6-transfected neurons (ANOVA, $P<0.005)$. Two-hundred-seventy-two neurons were analyzed. $(H)$ Granule neurons transfected with the TRPC5 RNAi or control U6 plasmid together with an expression plasmid encoding farnesylated GFP (fGFP) were subjected to immunocytochemistry using the GFP or phosphoSer51-Cdc20 antibody. The percentage of neurons with phosphoSer51-Cdc20 immunoreactivity was significantly reduced in TRPC5 knockdown neurons compared with control U6-transfected neurons (ANOVA, $P<0.005$ ). One-hundred-eighty-four neurons were analyzed. (I) Granule neurons transfected with the expression plasmid encoding TRPC5-WT, TRPC5- $\Delta \mathrm{N}$ term, or control vector together with the fGFP expression plasmid were analyzed as in $H$. The percentage of neurons with phosphoSer51-Cdc20 immunoreactivity was significantly increased in TRPC5-WT-expressing neurons, but not TRPC5- $\Delta \mathrm{N}$ term-expressing neurons, as compared with control vector-transfected neurons (ANOVA, $P<0.0001$ ). Five-hundred-eight-one neurons were analyzed. $(J)$ Granule neurons transfected with the TRPC5 expression plasmid or control vector together with the CaMKII $\beta$ RNAi or control U6 plasmid and the GFP expression plasmid were analyzed as in E. Expression of TRPC5 significantly reduced total dendrite length compared with control. CaMKII $\beta$ RNAi significantly increased total dendrite length in neurons in the presence or absence of TRPC5 expression (ANOVA, $P<0.0001)$. Three-hundred-sixty neurons were measured. $(K)$ Granule neurons transfected with the Cdc20 RNAi or control U6 plasmid together with the TRPC5-DN expression plasmid or control vector and the GFP expression plasmid were analyzed as in $E$. Expression of TRPC5-DN significantly increased total dendrite length compared with control. Cdc20 RNAi significantly reduced total dendrite length in neurons in the presence or absence of TRPC5-DN (ANOVA, $P<0.0001$ ). Three-hundred-sixty neurons were measured. $(L)$ Model of TRPC5 regulation of centrosomal CaMKII $\beta$ signaling in the control of dendrite patterning in the mammalian brain.

To determine whether the interaction of TRPC5 and CaMKII $\beta$ is important for the regulation of dendrite patterning, we expressed TRPC5-RES or TRPC5-RES lacking the $\mathrm{N}$-terminal cytosolic domain (TRPC5-RES
$\Delta \mathrm{N}$-term), which mediates the interaction of TRPC5 with CaMKII $\beta$, in the background of TRPC5 RNAi in granule neurons. In contrast to TRPC5-RES, which restricted dendrite elaboration and growth, TRPC5-RES $\Delta \mathrm{N}$-term 
had little or no effect on the TRPC5 RNAi-induced phenotype (Fig. 6E). These results suggest the TRPC5/ CaMKII $\beta$ interaction plays a critical role in the regulation of dendrite patterning.

To explore the role of TRPC5 in the regulation of CaMKII $\beta$ activity, we used a phosphoThr287-CaMKII antibody. Upon binding to calcium/CaM, СaMKII $\beta$ is autophosphorylated at Thr287, and thus phosphorylation at this site reflects activation of CaMKII $\beta$ (Miller and Kennedy 1986). TRPC5 knockdown, achieved by two distinct shRNAs, substantially reduced the phosphoThr287CaMKII immunoreactive signal in granule neurons (Fig. $6 \mathrm{~F}, \mathrm{G})$, suggesting that TRPC 5 stimulates the activation of CaMKII $\beta$ in neurons.

We next asked how TRPC5 activation of CaMKII $\beta$ might specifically regulate CaMKII $\beta$ signaling at the centrosome. Because PCM1 localizes CaMKII $\beta$ to the centrosome (Puram et al. 2011), we assessed the effect of autophosphorylation at Thr287 on the interaction of CaMKII $\beta$ with PCM1. We found that PCM1 interacted more efficiently with the autophosphorylation mimic T287D CaMKII $\beta$ mutant than wild-type CaMKII $\beta$ (Supplemental Fig. S7E). In contrast, PCM1 failed to associate with the loss of autophosphorylation (T287A) CaMKII $\beta$ mutant (data not shown). These results suggest that TRPC5 stimulates the autophosphorylation of CaMKII $\beta$, which in turn induces the interaction of activated CaMKII $\beta$ with the centrosomal targeting protein PCM1.

We next determined whether TRPC5 regulates CaMKI $\beta$ signaling at the centrosome. CaMKII $\beta$ phosphorylates the ubiquitin ligase coactivator Cdc20 at Ser51 in neurons, triggering Cdc20 dispersion from the centrosome and inhibiting the ubiquitin ligase activity of Cdc20-APC (Puram et al. 2011). Activation of TRPC5 with lanthanum chloride increased the number of neurons with Ser51phosphorylated Cdc20, whereas TRPC5 knockdown or inhibition with SKF96365 or flufenamic acid reduced the number of neurons with Ser51-phosphorylated Cdc20 (Fig. $6 \mathrm{H}$; Supplemental Fig. 7F; data not shown). Consistent with these results, immunoblotting analyses revealed reduced Ser51-phosphorylated Cdc20 in brain lysates of TRPC5 knockout animals compared with wild-type littermates (Supplemental Fig. S7G). In other experiments, expression of wild-type TRPC5, but not TRPC5 lacking the CaMKIIß-interacting N-terminal domain, induced Cdc20 phosphorylation at Ser51 (Fig. 6I), suggesting that the TRPC5/CaMKII $\beta$ interaction plays a critical role in TRPC5-induced Cdc20 phosphorylation. We also found that PCM1 knockdown abrogated lanthanum chloride-induced Cdc20 phosphorylation (Supplemental Fig. S7F), suggesting that PCM1 is required for TRPC5 activation of centrosomal CaMKII $\beta$ signaling.

Consistent with our results demonstrating that TRPC5 induces centrosomal CaMKII $\beta / \mathrm{Cdc} 20$ signaling, expression of TRPC5 increased Cdc20 dispersion from the centrosome in granule neurons, and CaMKII $\beta$ knockdown or PCM1 knockdown blocked TRPC5-induced dispersion of Cdc20 (Supplemental Fig. S7H). Finally, TRPC5 induced the dispersion of wild-type Cdc20 in neurons, but failed to significantly alter the centrosomal localization of a Cdc20 mutant in which Ser51 was replaced with alanine (S51A Cdc20) (Supplemental Fig. S7I). Taken together, our data suggest that TRPC5 stimulates CaMKII $\beta$ activity and triggers downstream signaling at the centrosome in neurons.

A prediction of these findings is that CaMKII $\beta$ signaling at the centrosome should operate downstream from TRPC5 in the regulation of dendrite morphogenesis. Consistent with this hypothesis, in epistasis analyses, CaMKII $\beta$ knockdown suppressed the ability of TRPC5 to restrict dendrite elaboration in granule neurons (Fig. 6J). In addition, expression of constitutively active T287D CaMKII $\beta$, but not the kinase-inactive T287A CaMKII $\beta$ mutant, suppressed the TRPC5 RNAi-induced dendrite phenotype (Supplemental Fig. S8A). In complementary analyses, expression of T287D CaMKII $\beta$ also suppressed the exuberant growth of dendrites in TRPC5 knockout granule neurons (Supplemental Fig. S8B). In other experiments, Cdc20 knockdown suppressed the ability of the dominant interfering form of TRPC5 (TRPC5-DN) to block dendrite retraction in neurons (Fig. 6K). These results suggest that CaMKII $\beta$ and Cdc20-APC operate downstream from TRPC5 in the regulation of dendrite morphogenesis. Collectively, our data define a novel function for TRPC5 that links calcium to activation of the centrosomal CaMKII $\beta / C d c 20-A P C$ pathway and consequently controls dendrite patterning in the mammalian brain (see model in Fig. 6L).

\section{Discussion}

In this study, we discovered a novel calcium signaling link that couples the TRP channel TRPC5 with the protein kinase CaMKII $\beta$ and thereby orchestrates dendrite morphogenesis and connectivity in the mammalian brain. Using independent genetic approaches of TRPC5 inhibition, we identified an essential, cell-autonomous function for TRPC5 in dendrite patterning in the mammalian brain in vivo. Correlating with its function in dendrite morphogenesis in the cerebellar cortex, TRPC5 appears to play a critical role in normal motor coordination and gait in mice. We also found that TRPC5 forms a specific complex with CaMKII $\beta$, but not CaMKII $\alpha$, and thus triggers the activation of CaMKII $\beta$, leading to the phosphorylation and inhibition of the major ubiquitin ligase Cdc20-APC at the centrosome. Activation of centrosomal CaMKII $\beta$ signaling mediates TRPC5-induced restriction of dendrite growth. Collectively, our findings define a novel TRPC5-dependent mechanism by which calcium signaling activates a centrosomal ubiquitin ligase pathway and thereby regulates dendrite patterning in the brain.

The finding that TRPC5 regulates dendrite patterning in the mammalian brain suggests that TRP channels may have important developmental functions in addition to their sensory receptive roles in mature neurons. The regulation of dendrite patterning by TRPC 5 in the cerebellar cortex correlates with a requirement for TRPC 5 in normal motor system function. In the future, it will be important 
to employ inducible and tissue-specific knockdown or knockout approaches to determine whether dendrite abnormalities in the cerebellar cortex upon TRPC5 inhibition lead to abnormalities in motor coordination and gait ataxia. In addition, it will be interesting to determine whether control of dendrite patterning by TRPC5 in other regions of the brain might play a role in other behaviors, including innate fear-induced responses (Riccio et al. 2009).

The identification of TRPC5 as the source of calcium that activates the isoform-specific function of CaMKII $\beta$ in dendrite patterning illuminates how extrinsic cues may regulate centrosomal signaling pathways dedicated to dendrite morphogenesis. Recent studies suggest that the centrosome represents a critical subcellular site for integration of signals that regulate dendrite development (Kim et al. 2009; Puram et al. 2011). However, prior to our study, it was unclear how calcium entry and extrinsic cues might regulate centrosomal pathways of dendrite development. Remarkably, we found that TRPC5 specifically associates with CaMIIK $\beta$, but not CaMKII $\alpha$, and thereby controls CaMKII $\beta$ signaling at the centrosome. The specific interaction of TRPC5 with CaMKII $\beta$ provides an interesting counterpoint to the interaction of CaMKII $\alpha$ with L-type VSCCs (Hudmon et al. 2005; Grueter et al. 2008). L-type VSCCs trigger CaMKII $\alpha$-dependent neuronal responses, including dendrite growth and elaboration (Gaudilliere et al. 2004), but fail to activate the centrosomal СaMKII $\beta$ signaling pathway (data not shown). In contrast, TRPC5 specifically promotes centrosomal CaMKII $\beta$ signaling, thereby restricting dendrite elaboration and growth. Together, these observations support the concept that protein-protein interactions between calcium channels and their effectors confer functional specificity through the activation of distinct intracellular signal transduction pathways in neurons.

The identification of TRPC5 as a channel that activates CaMKII $\beta$ also reveals a novel link between calcium entry and the ubiquitination machinery at the centrosome in neurons. In other subcellular locales, calcium entry via NMDA receptors has been associated with the redistribution of proteasomes from dendritic shafts to spines, and subsequent changes in protein degradation are thought to remodel spines and neuronal connectivity (Bingol and Schuman 2005, 2006; Mabb and Ehlers 2010). It will be interesting in future studies to explore whether TRPC5 regulates the ubiquitination machinery outside of the centrosome, including at dendritic spines.

The precise mechanism gating the TRPCs is controversial (Clapham 2003). G-protein-coupled receptors may activate TRPCs (Gee et al. 2003; Tozzi et al. 2003; Meis et al. 2007) via phospholipase $C$ through either inositol triphosphate or diacylglycerol (Schaefer et al. 2000; Clapham 2003). Other studies have suggested that TRPCs may be gated by calcium sensors such as stomal-interacting protein 1 (STIM1) and thereby function as store-operated channels (Baba et al. 2006; Yuan et al. 2007). In the future, it will be important to identify the critical upstream regulators of TRPC5 and determine whether they influence centrosomal CaMKII/Cdc20-APC signaling in the control of dendrite patterning in the mammalian brain.
Activation of TRPC5/CaMKII $\beta$ signaling drives an essential regulatory pathway that provides a counterpoint to dendrite growth and branching, thereby contributing to the careful balance that yields mature dendrite arbors in the mammalian brain. The question arises whether this signaling link is relevant to neurological diseases. Notably, abnormalities in dendrite development have been reported in diverse neurological diseases, including mental retardation and autism spectrum disorders (Kaufmann and Moser 2000; Dierssen and Ramakers 2006; Pardo and Eberhart 2007). TRPC5 is located within a region of the $\mathrm{X}$ chromosome that contains loci for nonsyndromic mental retardation (MRX47 and MRX35) (Gu et al. 1996; des Portes et al. 1997; Sossey-Alaoui et al. 1999). It will be of interest to explore the possibility that mutations in TRPC5 and deregulation of TRPC5-regulated CaMKII $\beta$ signaling might lead to defects in dendrite development and thereby contribute to neurodevelopmental diseases, including mental retardation and autism spectrum disorders.

\section{Materials and methods}

\section{Primary neuron cultures and transfection}

Primary cerebellar granule neurons were prepared from P6 rat pups and maintained in full medium (basal medium, Eagle [BME] plus $10 \%$ calf serum [Hyclone]; $1 \mathrm{mM}$ penicillin, streptomycin, L-glutamine; $25 \mathrm{mM} \mathrm{KCl}$ ). Neurons were transfected on DIV2 ( $2 \mathrm{~d}$ in vitro) using a modified calcium phosphate protocol as described (Konishi et al. 2004). Transfection of neurons with two plasmids encoding GFP and dsRed, respectively, led to coexpression of GFP and dsRed in all transfected neurons (data not shown). To avoid the possibility that morphological effects of RNAi or protein expression were a result of changes in cell survival, we included an expression plasmid encoding the antiapoptotic protein Bcl-xl in all neuronal transfections. As reported, expression of Bcl-xl had little or no effect on dendrite morphology (Gaudilliere et al. 2004, Tolias et al. 2005). TRPC5 restricted dendrite growth in the presence or absence of Bcl-xl (Supplemental Fig. S9).

TRPC5 knockout cultures and diolistic analyses of dendrite arbors in vivo

Primary cerebellar granule neurons prepared from P5 TRPC5 knockout mice and wild-type littermates were maintained in full medium (Riccio et al. 2009). Granule neuron dendrite arbors were visualized upon expression of GFP. Hippocampal neuron cultures were prepared from P1 TRPC5 knockout and wild-type littermates as described (Brewer 1995). For diolistic analyses of dendrite arbors in vivo, cerebellar and hippocampal sections were analyzed as described (Gan et al. 2000; Wu et al. 2004; O'Brien and Lummis 2006). Briefly, coronal sections of the cerebellum and hippocampus were fixed for $10 \mathrm{~min}$ in $4 \%$ paraformaldehyde and then incubated in $30 \%$ sucrose for $1 \mathrm{~h}$ at $4^{\circ} \mathrm{C}$. Sections were transferred and diolistically labeled with DiI-coated tungsten particles using a gene gun (Bio-Rad), then maintained in $4 \%$ paraformaldehyde overnight and mounted for analysis.

\section{Cerebellar slice cultures and in vivo electroporation}

P10 rat cerebella were prepared as described (Gaudilliere et al. 2004; Shalizi et al. 2006, 2007). Briefly, cerebella were dissected 
in HHGN (2.5 mM HEPES, $35 \mathrm{mM}$ glucose, $4 \mathrm{mM} \mathrm{NaHCO}$ diluted in Cellgro HBSS), sectioned sagitally using a tissue chopper (Mclllwain) into $400-\mu \mathrm{m}$ sections, and transferred onto a porous membrane (Millipore), allowing for an air-medium interface. Slices were maintained in serum-containing MEM. Individual neurons in P10 slices were transfected after $2 \mathrm{~d}$ using biolistics (Helios gene gun, Bio-Rad) as described (Gaudilliere et al. 2004; Shalizi et al. 2006). Four days after transfection, slices were subjected to immunohistochemical analyses.

All experiments using live animals have been approved by the Harvard Medical School Standing Committee on Animals. In vivo electroporation of P3 Sprague-Dawley rat pups was performed as described (Konishi et al. 2004). Five days or $9 \mathrm{~d}$ after electroporation (P8 or P12, respectively), animals were euthanized and cerebella were harvested. Coronal sections of cerebella $(40 \mu \mathrm{m})$ were prepared and subjected to immunohistochemistry with the GFP and Calbindin antibody and the DNA dye bisbenzimide (Hoechst 33258).

\section{Immunocytochemistry}

For visualization of centrosomal proteins, neurons were fixed in absolute methanol for $10 \mathrm{~min}$ at $-20^{\circ} \mathrm{C}$ and subjected to immunofluorescence analysis after blocking and staining with the indicated antibodies according to standard protocols. For other immunocytochemistry experiments, neurons were fixed in $4 \%$ paraformaldehyde for $20 \mathrm{~min}$ at room temperature and analyzed as described (Konishi et al. 2004). Cells were counted as having dispersed Cdc20 as described (Puram et al. 2011).

\section{Immunoprecipitation analyses}

Cells were lysed in $150 \mathrm{mM} \mathrm{NaCl}, 20 \mathrm{mM}$ Tris- $\mathrm{HCl}$ (pH 7.5), $1 \mathrm{mM}$ EDTA, and 1\% NP40 containing protease inhibitors. Lysates were briefly precleared with a combination of protein A/G sepharose beads and then incubated with either the appropriate antibody or antibody-conjugated beads overnight. For nonconjugated antibodies, the antibody-protein complexes were immunoprecipitated with protein $\mathrm{A} / \mathrm{G}$ beads. Immunoprecipitated proteins bound to beads were washed several times, and lysates were analyzed by SDS-PAGE and transferred to a nitrocellulose membrane for immunoblotting analysis.

\section{Analysis of neuronal morphology and imaging}

To analyze the axonal and dendritic morphology of primary neurons in culture, in slices, and in vivo, images of individual neurons were captured randomly in a blinded manner on a Nikon Eclipse TE2000 epifluorescence microscope using a digital CCD camera (Diagnostic Instruments). SPOT software was used to measure individual process length by tracing. Axons and dendrites were identified in transfected neurons based on morphology and selective expression of MAP2 and Taul in dendrites and axons, respectively (data not shown). Total dendrite length was determined by summing the lengths of all dendrite processes measured from a single neuron. To analyze dendrite morphology in vivo, granule neurons residing in the IGL were selected for morphometry.

Confocal images were collected using an Olympus IX81 microscope with a FluoView1000 scanning confocal unit /taken with a $40 \times / 0.90$ NA Olympus UPlanSApo or $60 \times / 1.42$ NA Oil Olympus PlanApoN objective). Labeled neurons were excited at $405 \mathrm{~nm}, 488 \mathrm{~nm}$, and $559 \mathrm{~nm}$, and emission was collected at 425$475 \mathrm{~nm}, 500-545 \mathrm{~nm}$, and 575-675 nm for Hoechst, Alexa Fluor488 , and Cy3, respectively.

\section{Behavioral analyses of motor coordination}

All behavioral tests and analyses were performed in a blinded manner using 4-mo-old, male wild-type and TRPC5 knockout littermates. Balance beam tests of motor coordination were performed as described (Carter et al. 1999). Mice were trained to walk on a wide $(20 \mathrm{~mm}$ width $\times 0.75 \mathrm{~m}$ length) balance beam for three trials. All mice traversed the wide beam without foot slips. Mice were then trained on a narrow $(4 \mathrm{~mm}$ width $\times 0.75 \mathrm{~m}$ length) beam for three trials. Mice were videotaped as they performed three test trials of three beam walks, for a total of nine runs per animal. Videotaped walks were scored for number of foot slips and time to cross.

Gait parameters were measured using the automated Digigait analysis system (Mouse Specifics). Using this system, mice were imaged ventrally with a high-frame-rate camera while running on a transparent treadmill. Software analysis was used to identify individual paw prints and calculate gait metrics based on the position, area, and timing of paw steps. All mice were run at $24 \mathrm{~cm} / \mathrm{sec}$.

\section{Acknowledgments}

We thank Caleb Yeung for technical assistance, and Dr. David Clapham and members of the Bonni laboratory for helpful discussions and critical reading of the manuscript. This work was supported by the National Institutes of Health grant NS051255 (to A.B.), a Human Frontier Science Program Longterm Fellowship (to Y.I.), and a Ruth L. Kirschstein National Research Service Award (National Cancer Institute) and a Brain Science Foundation grant (to A.H.K.).

\section{References}

Altman J, Bayer S. 1997. Development of the cerebellar system: In relation to its evolution, structure, and functions. CRC Press, New York.

Baba Y, Hayashi K, Fujii Y, Mizushima A, Watarai H, Wakamori M, Numaga T, Mori Y, Iino M, Hikida M, et al. 2006. Coupling of STIM1 to store-operated $\mathrm{Ca}^{2+}$ entry through its constitutive and inducible movement in the endoplasmic reticulum. Proc Natl Acad Sci 103: 16704-16709.

Bingol B, Schuman EM. 2005. Synaptic protein degradation by the ubiquitin proteasome system. Curr Opin Neurobiol 15: 536-541.

Bingol B, Schuman EM. 2006. Activity-dependent dynamics and sequestration of proteasomes in dendritic spines. Nature 441: $1144-1148$.

Brewer GJ. 1995. Serum-free B27/neurobasal medium supports differentiated growth of neurons from the striatum, substantia nigra, septum, cerebral cortex, cerebellum, and dentate gyrus. I Neurosci Res 42: 674-683.

Carter RJ, Lione LA, Humby T, Mangiarini L, Mahal A, Bates GP, Dunnett SB, Morton AJ. 1999. Characterization of progressive motor deficits in mice transgenic for the human Huntington's disease mutation. J Neurosci 19: 3248-3257.

Clapham DE. 2003. TRP channels as cellular sensors. Nature 426: 517-524.

Cline H, Haas K. 2008. The regulation of dendritic arbor development and plasticity by glutamatergic synaptic input: A review of the synaptotrophic hypothesis. J Physiol 586: 1509-1517.

Davare MA, Fortin DA, Saneyoshi T, Nygaard S, Kaech S, Banker G, Soderling TR, Wayman GA. 2009. Transient receptor potential canonical 5 channels activate $\mathrm{Ca}^{2+} / \mathrm{cal}$ modulin kinase I $\gamma$ to promote axon formation in hippocampal neurons. J Neurosci 29: 9794-9808. 
de la Torre-Ubieta L, Bonni A. 2011. Transcriptional regulation of neuronal polarity and morphogenesis in the mammalian brain. Neuron 72: 22-40.

de la Torre-Ubieta L, Gaudilliere B, Yang Y, Ikeuchi Y, Yamada T, DiBacco S, Stegmuller J, Schuller U, Salih DA, Rowitch D, et al. 2010. A FOXO-Pak1 transcriptional pathway controls neuronal polarity. Genes Dev 24: 799-813.

des Portes V, Soufir N, Carrie A, Billuart P, Bienvenu T, Vinet MC, Beldjord C, Ponsot G, Kahn A, Boue J, et al. 1997. Gene for nonspecific X-linked mental retardation (MRX 47) is located in Xq22.3-q24. Am J Med Genet 72: 324-328.

Dierssen M, Ramakers GJ. 2006. Dendritic pathology in mental retardation: From molecular genetics to neurobiology. Genes Brain Behav 5: 48-60.

Ebersbach G, Sojer M, Valldeoriola F, Wissel J, Muller J, Tolosa E, Poewe W. 1999. Comparative analysis of gait in Parkinson's disease, cerebellar ataxia and subcortical arteriosclerotic encephalopathy. Brain 122: 1349-1355.

Gan WB, Grutzendler J, Wong WT, Wong RO, Lichtman JW. 2000. Multicolor 'DiOlistic' labeling of the nervous system using lipophilic dye combinations. Neuron 27: 219-225.

Gaudilliere B, Shi Y, Bonni A. 2002. RNA interference reveals a requirement for myocyte enhancer factor $2 \mathrm{~A}$ in activitydependent neuronal survival. J Biol Chem 277: 46442-46446.

Gaudilliere B, Konishi Y, de la Iglesia N, Yao G, Bonni A. 2004. A CaMKII-NeuroD signaling pathway specifies dendritic morphogenesis. Neuron 41: 229-241.

Gee CE, Benquet P, Gerber U. 2003. Group I metabotropic glutamate receptors activate a calcium-sensitive transient receptor potential-like conductance in rat hippocampus. J Physiol 546: 655-664.

Greka A, Navarro B, Oancea E, Duggan A, Clapham DE. 2003. TRPC5 is a regulator of hippocampal neurite length and growth cone morphology. Nat Neurosci 6: 837-845.

Grueber WB, Jan YN. 2004. Dendritic development: Lessons from Drosophila and related branches. Curr Opin Neurobiol 14: $74-82$.

Grueter CE, Abiria SA, Wu Y, Anderson ME, Colbran RJ. 2008. Differential regulated interactions of calcium/calmodulindependent protein kinase II with isoforms of voltage-gated calcium channel $\beta$ subunits. Biochemistry 47: 1760-1767.

Gu XX, Decorte R, Marynen P, Fryns JP, Cassiman JJ, Raeymaekers P. 1996. Localisation of a new gene for nonspecific mental retardation to Xq22-q26 (MRX35). I Med Genet 33: 52-55.

Hamori J, Somogyi J. 1983. Differentiation of cerebellar mossy fiber synapses in the rat: A quantitative electron microscope study. J Comp Neurol 220: 365-377.

Hatten ME, Heintz N. 1995. Mechanisms of neural patterning and specification in the developing cerebellum. Annu Rev Neurosci 18: 385-408.

Hausser M, Spruston N, Stuart GJ. 2000. Diversity and dynamics of dendritic signaling. Science 290: 739-744.

Hudmon A, Schulman H, Kim J, Maltez JM, Tsien RW, Pitt GS. 2005. CaMKII tethers to L-type $\mathrm{Ca}^{2+}$ channels, establishing a local and dedicated integrator of $\mathrm{Ca}^{2+}$ signals for facilitation. J Cell Biol 171: 537-547.

Hurlock EC, Bose M, Pierce G, Joho RH. 2009. Rescue of motor coordination by Purkinje cell-targeted restoration of Kv3.3 channels in Kcnc3-null mice requires Kcnc1. J Neurosci 29: 15735-15744.

Jan YN, Jan LY. 2003. The control of dendrite development. Neuron 40: 229-242.

Kanaseki T, Ikeuchi Y, Sugiura H, Yamauchi T. 1991. Structural features of $\mathrm{Ca}^{2+} /$ calmodulin-dependent protein kinase II revealed by electron microscopy. J Cell Biol 115: 1049-1060.
Kaufmann WE, Moser HW. 2000. Dendritic anomalies in disorders associated with mental retardation. Cereb Cortex 10: 981-991.

Kim AH, Puram SV, Bilimoria PM, Ikeuchi Y, Keough S, Wong M, Rowitch D, Bonni A. 2009. A centrosomal Cdc20-APC pathway controls dendrite morphogenesis in postmitotic neurons. Cell 136: 322-336.

Konishi Y, Stegmuller J, Matsuda T, Bonni S, Bonni A. 2004. Cdh1-APC controls axonal growth and patterning in the mammalian brain. Science 303: 1026-1030.

Konur S, Ghosh A. 2005. Calcium signaling and the control of dendritic development. Neuron 46: 401-405.

Kravitz AV, Freeze BS, Parker PR, Kay K, Thwin MT, Deisseroth K, Kreitzer AC. 2010. Regulation of parkinsonian motor behaviours by optogenetic control of basal ganglia circuitry. Nature 466: $622-626$.

Mabb AM, Ehlers MD. 2010. Ubiquitination in postsynaptic function and plasticity. Annu Rev Cell Dev Biol 26: 179-210.

McKemy DD, Neuhausser WM, Julius D. 2002. Identification of a cold receptor reveals a general role for TRP channels in thermosensation. Nature 416: 52-58.

Meis S, Munsch T, Sosulina L, Pape HC. 2007. Postsynaptic mechanisms underlying responsiveness of amygdaloid neurons to cholecystokinin are mediated by a transient receptor potential-like current. Mol Cell Neurosci 35: 356-367.

Miller SG, Kennedy MB. 1985. Distinct forebrain and cerebellar isozymes of type II $\mathrm{Ca}^{2+}$ /calmodulin-dependent protein kinase associate differently with the postsynaptic density fraction. J Biol Chem 260: 9039-9046.

Miller SG, Kennedy MB. 1986. Regulation of brain type II Ca ${ }^{2+} /$ calmodulin-dependent protein kinase by autophosphorylation: A Ca ${ }^{2+}$-triggered molecular switch. Cell 44: 861-870.

Montell C, Birnbaumer L, Flockerzi V. 2002. The TRP channels, a remarkably functional family. Cell 108: 595-598.

O'Brien JA, Lummis SC. 2006. Diolistic labeling of neuronal cultures and intact tissue using a hand-held gene gun. Nat Protoc 1: 1517-1521.

Palay S, Chan-Palay V. 1974. Cerebellar cortex: Cytology and organization. Springer-Verlag, New York.

Palliyath S, Hallett M, Thomas SL, Lebiedowska MK. 1998. Gait in patients with cerebellar ataxia. Mov Disord 13: 958-964.

Pardo CA, Eberhart CG. 2007. The neurobiology of autism. Brain Pathol 17: 434-447.

Puram SV, Kim AH, Ikeuchi Y, Wilson-Grady JT, Merdes A, Gygi SP, Bonni A. 2011. A CaMKII $\beta$ signaling pathway at the centrosome regulates dendrite patterning in the brain. Nat Neurosci 14: 973-983.

Rajan I, Witte S, Cline HT. 1999. NMDA receptor activity stabilizes presynaptic retinotectal axons and postsynaptic optic tectal cell dendrites in vivo. J Neurobiol 38: 357-368.

Ramon y Cajal S. 1995. Histology of the nervous system of man and vertebrates (ed. N Swanson, L Swanson). Oxford University Press, New York.

Ramsey IS, Delling M, Clapham DE. 2006. An introduction to TRP channels. Annu Rev Physiol 68: 619-647.

Redmond L, Kashani AH, Ghosh A. 2002. Calcium regulation of dendritic growth via CaM kinase IV and CREB-mediated transcription. Neuron 34: 999-1010.

Riccio A, Li Y, Moon J, Kim KS, Smith KS, Rudolph U, Gapon S, Yao GL, Tsvetkov E, Rodig SI, et al. 2009. Essential role for TRPC5 in amygdala function and fear-related behavior. Cell 137: 761-772.

Schaefer M, Plant TD, Obukhov AG, Hofmann T, Gudermann T, Schultz G. 2000. Receptor-mediated regulation of the nonselective cation channels TRPC4 and TRPC5. I Biol Chem 275: $17517-17526$. 
Shalizi A, Gaudilliere B, Yuan Z, Stegmuller J, Shirogane T, Ge Q, Tan Y, Schulman B, Harper JW, Bonni A. 2006. A calciumregulated MEF2 sumoylation switch controls postsynaptic differentiation. Science 311: 1012-1017.

Shalizi A, Bilimoria PM, Stegmuller J, Gaudilliere B, Yang Y, Shuai K, Bonni A. 2007. PIASx is a MEF2 SUMO E3 ligase that promotes postsynaptic dendritic morphogenesis. J Neurosci 27: 10037-10046.

Sin WC, Haas K, Ruthazer ES, Cline HT. 2002. Dendrite growth increased by visual activity requires NMDA receptor and Rho GTPases. Nature 419: 475-480.

Sossey-Alaoui K, Lyon JA, Jones L, Abidi FE, Hartung AJ, Hane B, Schwartz CE, Stevenson RE, Srivastava AK. 1999. Molecular cloning and characterization of TRPC5 (HTRP5), the human homologue of a mouse brain receptor-activated capacitative $\mathrm{Ca}^{2+}$ entry channel. Genomics 60: 330-340.

Talavera K, Nilius B, Voets T. 2008. Neuronal TRP channels: Thermometers, pathfinders and life-savers. Trends Neurosci 31: 287-295.

Tolias KF, Bikoff JB, Burette A, Paradis S, Harrar D, Tavazoie S, Weinberg RJ, Greenberg ME. 2005. The Rac1-GEF Tiam1 couples the NMDA receptor to the activity-dependent development of dendritic arbors and spines. Neuron 45: 525538.

Tozzi A, Bengtson CP, Longone P, Carignani C, Fusco FR, Bernardi G, Mercuri NB. 2003. Involvement of transient receptor potential-like channels in responses to mGluR-I activation in midbrain dopamine neurons. Eur J Neurosci 18: 2133-2145.

Vaillant AR, Zanassi P, Walsh GS, Aumont A, Alonso A, Miller FD. 2002. Signaling mechanisms underlying reversible, activitydependent dendrite formation. Neuron 34: 985-998.

Vallano ML. 1989. Separation of isozymic forms of type II calcium/calmodulin-dependent protein kinase using cationexchange chromatography. I Neurosci Methods 30: 1-9.

Wong RO, Ghosh A. 2002. Activity-dependent regulation of dendritic growth and patterning. Nat Rev Neurosci 3: 803812.

Wu GY, Cline HT. 1998. Stabilization of dendritic arbor structure in vivo by CaMKII. Science 279: 222-226.

Wu CC, Reilly JF, Young WG, Morrison JH, Bloom FE. 2004. High-throughput morphometric analysis of individual neurons. Cereb Cortex 14: 543-554.

Yang Y, Kim AH, Bonni A. 2010. The dynamic ubiquitin ligase duo: Cdh1-APC and Cdc20-APC regulate neuronal morphogenesis and connectivity. Curr Opin Neurobiol 20: 92-99.

Yuan JP, Zeng W, Huang GN, Worley PF, Muallem S. 2007. STIM1 heteromultimerizes TRPC channels to determine their function as store-operated channels. Nat Cell Biol 9: 636-645. 


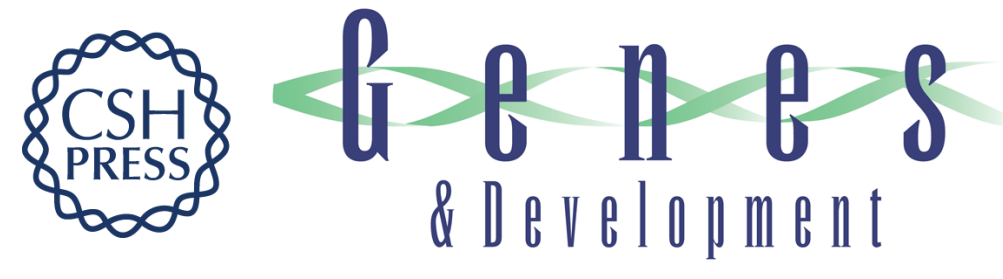

\section{A TRPC5-regulated calcium signaling pathway controls dendrite patterning in the mammalian brain}

Sidharth V. Puram, Antonio Riccio, Samir Koirala, et al.

Genes Dev. 2011, 25: originally published online December 1, 2011

Access the most recent version at doi:10.1101/gad.174060.111

\section{Supplemental http://genesdev.cshlp.org/content/suppl/2011/12/02/gad.174060.111.DC1 Material}

References This article cites 62 articles, 16 of which can be accessed free at: http://genesdev.cshlp.org/content/25/24/2659.full.html\#ref-list-1

\section{License}

Email Alerting

Service

Receive free email alerts when new articles cite this article - sign up in the box at the top right corner of the article or click here.

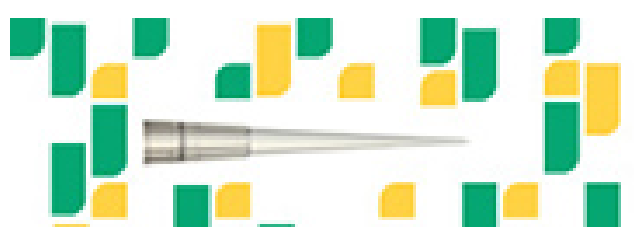

Focused on your science. 